\title{
O'Neil Inequality for Convolutions Associated with Gegenbauer Differential Operator and some Applications
}

\author{
Vagif S. Guliyev $^{1,2, *}$, E.J. Ibrahimov ${ }^{2}$, S.E. Ekincioglu ${ }^{1}$ \\ and S. Ar. Jafarova ${ }^{3}$ \\ ${ }^{1}$ Department of Mathematics, Dumlupinar University, Kutahya, Turkey; \\ ${ }^{2}$ Institute of Mathematics and Mechanics of NASA, AZ 1141 Baku, Azerbaijan; \\ ${ }^{3}$ Azerbaijan State Economic University, AZ 1001, Baku, Azerbaijan.
}

Received April 8, 2019; Accepted September 2, 2019;

Published online March 4, 2020

\begin{abstract}
In this paper we prove an $\mathrm{O}^{\prime} \mathrm{Neil}$ inequality for the convolution operator (G-convolution) associated with the Gegenbauer differential operator $G_{\lambda}$. By using an $\mathrm{O}^{\prime}$ Neil inequality for rearrangements we obtain a pointwise rearrangement estimate of the G-convolution. As an application, we obtain necessary and sufficient conditions on the parameters for the boundedness of the $G$-fractional maximal and $G$-fractional integral operators from the spaces $L_{p, \lambda}$ to $L_{q, \lambda}$ and from the spaces $L_{1, \lambda}$ to the weak spaces $W L_{p, \lambda}$.
\end{abstract}

AMS subject classifications: 42B20, 42B25, 42B35, 47G10, 47B37

Key words: Gegenbauer differential operator, G-convolution, O’Neil inequality, G-fractional integral, G-fractional maximal function.

\section{Introduction}

For $1 \leq p \leq \infty$, let $L_{p, \lambda}\left(\mathbb{R}_{+}, s h^{2 \lambda} x d x\right)$ be the spaces of measurable functions on $\mathbb{R}_{+}=$ $(0, \infty)$ with the finite norm

$$
\begin{aligned}
& \|f\|_{L_{p, \lambda}\left(\mathbb{R}_{+}\right)}=\left(\int_{\mathbb{R}_{+}}|f(\operatorname{ch} x)|^{p} \operatorname{sh}^{2 \lambda} x d x\right)^{\frac{1}{p}}, \quad 1 \leq p<\infty, \\
& \|f\|_{L_{\infty, \lambda}} \equiv\|f\|_{L_{\infty}\left(\mathbb{R}_{+}\right)}=\underset{x \in \mathbb{R}_{+}}{\operatorname{esssup}}|f(\operatorname{ch} x)|,
\end{aligned}
$$

*Corresponding author. Email addresses: vagif@guliyev.com (V. S. Guliyev), elmanibrahimov@yahoo.com (E. Ibrahimov), elifnurekincioglu@gmail.com (S. E. Ekincioglu), sada-jafarova@rambler.ru (S. Jafarova) 
where $0<\lambda<\frac{1}{2}$ is a fixed parameter.

Denote by $A_{\text {cht }}^{\lambda}$ the shift operator (G-shift) (see [9])

$$
A_{\text {cht }}^{\lambda} f(\operatorname{ch} x)=C_{\lambda} \int_{0}^{\pi} f(\operatorname{ch} x \operatorname{ch} t-\operatorname{sh} x \operatorname{sh} t \cos \varphi)(\sin \varphi)^{2 \lambda-1} d \varphi,
$$

generated by Gegenbauer differential operator $G_{\lambda}$

$$
G \equiv G_{\lambda}=\left(x^{2}-1\right)^{\frac{1}{2}-\lambda} \frac{d}{d x}\left(x^{2}-1\right)^{\lambda+\frac{1}{2}} \frac{d}{d x}, x \in(1, \infty), \lambda \in\left(0, \frac{1}{2}\right),
$$

where

$$
C_{\lambda}=\frac{\Gamma\left(\lambda+\frac{1}{2}\right)}{\Gamma(\lambda) \Gamma\left(\frac{1}{2}\right)}=\left(\int_{0}^{\pi}(\sin \varphi)^{2 \lambda-1} d \varphi\right)^{-1} .
$$

The Gegenbauer differential operator was introduced in [5]. For the properties of the Gegenbauer differential operator, we refer to [3,4,10-12].

The shift operator $A_{\text {cht }}^{\lambda}$ generates the corresponding convolution (G-convolution)

$$
(f \oplus g)(\operatorname{ch} x)=\int_{\mathbb{R}_{+}} f(\operatorname{ch} t) A_{\text {cht }}^{\lambda} g(\operatorname{ch} x) \operatorname{sh}^{2 \lambda} t d t .
$$

The paper is organized as follows. In Section 2, we give some results needed to facilitate the proofs of our theorems. In Section 3, we show that an $\mathrm{O}^{\prime}$ Neil inequality for rearrangements of the G-convolution holds. In Section 4, we prove an O'Neil inequality for G-convolution. In Section 5, we prove the boundedness of $G$-fractional maximal and $G$-fractional integral operators from the spaces $L_{p, \lambda}$ to $L_{q, \lambda}$ and from the spaces $L_{1, \lambda}$ to the weak spaces $W L_{q, \lambda}$. We show that the conditions on the boundedness cannot be weakened.

Further $A \lesssim B$ denotes that exists the constant $C>0$ such that $0<A \leq C B$, moreover $C$ can depend on some parameters. Symbol $A \approx B$ denote that $A \lesssim B$ and $B \lesssim A$.

\section{Some auxiliary results}

In this section we formulate some lemmas that will be needed later.

Lemma 2.1. 1) Let $1 \leq p \leq \infty, f \in L_{p, \lambda}\left(\mathbb{R}_{+}\right)$, then for all $t \in \mathbb{R}_{+}$

$$
\left\|A_{c h t}^{\lambda} f\right\|_{L_{p, \lambda}} \leq\|f\|_{L_{p, \lambda}} \text {. }
$$

2) Let $1 \leq p, r \leq q \leq \infty, \frac{1}{p^{\prime}}+\frac{1}{q^{\prime}}=\frac{1}{r}, p p^{\prime}=p+p^{\prime}, f \in L_{p, \lambda}\left(\mathbb{R}_{+}\right), g \in L_{r, \lambda}\left(\mathbb{R}_{+}\right)$. Then $f \oplus g \in$ $L_{q, \lambda}\left(\mathbb{R}_{+}\right)$and

$$
\|f \oplus g\|_{L_{q, \lambda}} \leq\|f\|_{L_{p, \lambda}}\|g\|_{L_{r, \lambda}}
$$

(see [9], Lemmas 2 and 4). 
For all measurable set $E \subset[0, \infty), \mu E=|E|_{\lambda}=\int_{E} s h^{2 \lambda} t d t$. We denote $H(x, r)=(x-r, x+$ $r) \cap \mathbb{R}_{+}$, i.e.,

$$
\begin{aligned}
& H(x, r)=\left\{\begin{array}{cc}
(0, x+r), & 0 \leq x<r, \\
(x-r, x+r), & r \leq x<\infty,
\end{array} \quad H(0, r)=(0, r),\right. \\
& |H(x, r)|_{\lambda}=\int_{H(x, r)} s h^{2 \lambda} t d t, \quad|H(0, r)|_{\lambda}=\int_{0}^{r} s h^{2 \lambda} t d t .
\end{aligned}
$$

Lemma 2.2. For any measurable $E \subset \mathbb{R}_{+}$the following relation holds

$$
\int_{E} A_{\text {cht }} f(\operatorname{ch} x) \operatorname{sh}^{2 \lambda} t d t \approx \int_{H(x, r)} f(\operatorname{chu}) \operatorname{sh}^{2 \lambda} u d u,
$$

where $r=\sup E$.

Proof. First we prove that

$$
\int_{0}^{\infty} A_{c h t} f(\operatorname{ch} x) \operatorname{sh}^{2 \lambda} t d t=\int_{0}^{\infty} f(\operatorname{ch} u) \operatorname{sh}^{2 \lambda} u d u .
$$

Indeed

$$
\begin{aligned}
& \int_{0}^{\infty} A_{\text {cht }} f(\text { ch } x) s h^{2 \lambda} t d t=\int_{1}^{\infty} A_{t} f(x)\left(t^{2}-1\right)^{\lambda-\frac{1}{2}} d t \\
= & C_{\lambda} \int_{1}^{\infty}\left(\int_{0}^{\pi} f\left(x t-\sqrt{x^{2}-1} \sqrt{t^{2}-1} \cos \varphi\right)(\sin \varphi)^{2 \lambda-1} d \varphi\right)\left(t^{2}-1\right)^{\lambda-\frac{1}{2}} d t .
\end{aligned}
$$

Making the substitution $z=x t-\sqrt{x^{2}-1} \sqrt{t^{2}-1} \cos \varphi$, we get

$$
\begin{aligned}
& \cos \varphi=(x t-z)\left(x^{2}-1\right)^{-\frac{1}{2}}\left(t^{2}-1\right)^{-\frac{1}{2}} d \varphi=\left(1-x^{2}-t^{2}-z^{2}+2 x t z\right)^{-\frac{1}{2}} d z \\
& (\sin \varphi)^{2 \lambda-1}=\left(1-x^{2}-t^{2}-z^{2}+2 x t r\right)^{\lambda-\frac{1}{2}}\left(x^{2}-1\right)^{\frac{1}{2}-\lambda}\left(t^{2}-1\right)^{\frac{1}{2}-\lambda} .
\end{aligned}
$$

Then we have

$$
\begin{aligned}
& \int_{1}^{\infty} A_{t} f(x)\left(t^{2}-1\right)^{\lambda-\frac{1}{2}} d t=C_{\lambda}\left(t^{2}-1\right)^{\frac{1}{2}-\lambda} \\
& \times \int_{1}^{\infty}\left(\int_{x t-\sqrt{x^{2}-1} \sqrt{t^{2}-1}}^{x t+\sqrt{x^{2}-1} \sqrt{t^{2}-1}}\left(1-x^{2}-t^{2}-z^{2}+2 x t z\right)^{\lambda-1} f(z) d z\right) d x
\end{aligned}
$$

Since $x t-\sqrt{x^{2}-1} \sqrt{t^{2}-1} \leq z \leq x t+\sqrt{x^{2}-1} \sqrt{t^{2}-1}$

$$
\begin{aligned}
& \Leftrightarrow|z-x t| \leq \sqrt{x^{2}-1} \sqrt{t^{2}-1} \Leftrightarrow z^{2}-2 x t z+x^{2} t^{2} \leq x^{2} t^{2}-x^{2}-t^{2}+1 \\
& \Leftrightarrow z^{2}-2 x t z \leq 1-x^{2}-t^{2} \Leftrightarrow x^{2}-2 x t z+z^{2} t^{2} \leq 1-z^{2}-t^{2}+z^{2} t^{2} \\
& \Leftrightarrow(x-z t)^{2} \leq\left(z^{2}-1\right)\left(t^{2}-1\right) \Leftrightarrow|x-z t| \leq \sqrt{z^{2}-1} \sqrt{t^{2}-1} \\
& \Leftrightarrow z t-\sqrt{z^{2}-1} \sqrt{t^{2}-1} \leq z t+\sqrt{z^{2}-1} \sqrt{t^{2}-1}
\end{aligned}
$$


and

$$
1 \leq z t-\sqrt{z^{2}-1} \sqrt{t^{2}-1} \leq x \leq z t+\sqrt{z^{2}-1} \sqrt{t^{2}-1}<\infty,
$$

then by changing the order of integration we obtain

$$
\begin{aligned}
& \int_{1}^{\infty} A_{t} f(x)\left(t^{2}-1\right)^{\lambda-\frac{1}{2}} d t=C_{\lambda}\left(t^{2}-1\right)^{\frac{1}{2}-\lambda} \\
& \times \int_{1}^{\infty}\left(\int_{z t-\sqrt{z^{2}-1} \sqrt{t^{2}-1}}^{z t+\sqrt{z^{2}-1} \sqrt{t^{2}-1}}\left(1-x^{2}-t^{2}-z^{2}+2 x t z\right)^{\lambda-1} d x\right) f(z) d z .
\end{aligned}
$$

On the other hand

$$
1-x^{2}-t^{2}-z^{2}+2 x t z=\left(z t+\sqrt{z^{2}-1} \sqrt{t^{2}-1}-x\right)\left(x-z t+\sqrt{z^{2}-1} \sqrt{t^{2}-1}\right) .
$$

Then use of the formula (see [2], p. 299)

$$
\int_{a}^{b}(x-a)^{\mu-1}(b-x)^{v-1} d x=(b-a)^{\mu+v-1} \frac{\Gamma(\mu) \Gamma(v)}{\Gamma(\mu+v)}
$$

by $\mu=v=\lambda$, we have

$$
\begin{aligned}
& \int_{1}^{\infty} A_{t} f(x)\left(t^{2}-1\right)^{\lambda-\frac{1}{2}} d t \\
= & C_{\lambda} 2^{2 \lambda-1} \frac{\Gamma^{2}(\lambda)}{\Gamma(2 \lambda)} \int_{1}^{\infty}\left(z^{2}-1\right)^{\lambda-\frac{1}{2}} f(z) d z=\int_{1}^{\infty} f(z)\left(z^{2}-1\right)^{\lambda-\frac{1}{2}} d z,
\end{aligned}
$$

since (see [2], p. 952) $\Gamma(2 \lambda)=\frac{2^{2 \lambda-1} \Gamma(\lambda) \Gamma\left(\lambda+\frac{1}{2}\right)}{\Gamma\left(\frac{1}{2}\right)}$.

Further we have

$$
\begin{aligned}
& \int_{E} A_{c h t} f(\operatorname{ch} x) \operatorname{sh}^{2 \lambda} t d t \\
= & \int_{0}^{\infty} A_{\text {cht }} f(\operatorname{ch} x) \chi_{E}(\operatorname{cht} t) \operatorname{sh}^{2 \lambda} t d t=\int_{0}^{\infty} f(\operatorname{cht} t) A_{\operatorname{cht}} \chi_{E}(\operatorname{ch} x) \operatorname{sh}^{2 \lambda} t d t,
\end{aligned}
$$

where $\chi_{E}$-characteristic function of the set $E \subset \mathbb{R}_{+}$, and also

$$
\int_{H(x, r)} f(c h u) \operatorname{sh}^{2 \lambda} u d u=\int_{0}^{\infty} f(\operatorname{ch} u) \chi_{H(x, r)}(\operatorname{ch} u) \operatorname{sh}^{2 \lambda} u d u .
$$

Now we prove that from (2.2) and (2.3) the assertion of lemma follows, i.e.,

$$
\begin{aligned}
\int_{0}^{\infty} f(\operatorname{cht} t) A_{\operatorname{ch} t} \chi_{E}(\operatorname{ch} x) \operatorname{sh}^{2 \lambda} t d t & \approx \int_{0}^{\infty} f(\operatorname{ch} u) \chi_{H(x, r)}(\operatorname{ch} u) \operatorname{sh}^{2 \lambda} u d u \\
& \Leftrightarrow A_{\operatorname{cht}} \chi_{E}(\operatorname{ch} x) \approx \chi_{H(x, r)}(\operatorname{ch} t) .
\end{aligned}
$$

Indeed

$$
A_{\text {cht }} \chi_{E}(\operatorname{ch} x)=C_{\lambda} \int_{0}^{\pi} \chi_{E}(x, t)_{\varphi}(\operatorname{sh} \varphi)^{2 \lambda-1} d \varphi,
$$


where $(x, t)_{\varphi}=\operatorname{ch} x \operatorname{cht}-\operatorname{sh} x \operatorname{sht} \cos \varphi$, and

$$
\chi_{E}(x, t)_{\varphi}= \begin{cases}1, & \text { if }(x, t)_{\varphi} \in E, \\ 0, & \text { if }(x, t)_{\varphi} \notin E .\end{cases}
$$

Let $r=\sup E$. Since $|x-t| \leq \operatorname{ch}(x-t) \leq(x, t)_{\varphi} \leq \operatorname{ch}(x+t)$, then $|x-t|>r \Rightarrow(x, t)_{\varphi}>r$. Therefore, from $|x-t|>r$ it follows that $A_{\text {cht }} \chi_{E}(\operatorname{ch} x)=0$.

In this way we obtain

$$
A_{\text {cht }} \chi_{E}(\operatorname{ch} x)=C_{\lambda} \int_{\left\{\varphi \in[0, \pi]:(x, t)_{\varphi}<r\right\}}(\sin \varphi)^{2 \lambda-1} d \varphi=A(x, t, r) .
$$

Taking in (2.4) $\cos \varphi=y$, we obtain

$$
A(x, t, r)=C_{\lambda} \int_{\varphi(x, t, r)}^{1}\left(1-y^{2}\right)^{\lambda-1} d y, \quad \text { where } \varphi(x, t, r)=\frac{\operatorname{ch} x \operatorname{ch} t-r}{\operatorname{sh} x \operatorname{sh} t} .
$$

Since $\operatorname{ch}(x-t) \leq r \leq \operatorname{ch}(x+t)$ then we have $-1 \leq \varphi(x, t, r) \leq 1$. Therefore we have

$$
A(x, t, r)=C_{\lambda} \int_{\varphi(x, t, r)}^{1}\left(1-y^{2}\right)^{\lambda-1} d y \leq C_{\lambda} \int_{-1}^{1}\left(1-y^{2}\right)^{\lambda-1} d y=1 .
$$

Let $-1 \leq \varphi(x, t, r) \leq 0$. Then

$$
\begin{aligned}
A(x, t, r) & =C_{\lambda} \int_{\varphi(x, t, r)}^{1}\left(1-y^{2}\right)^{\lambda-1} d y \geq C_{\lambda} \int_{0}^{1}\left(1-y^{2}\right)^{\lambda-1} d y \\
& \geq C_{\lambda} 2^{\lambda-1} \int_{0}^{1}(1-y)^{\lambda-1} d y=\frac{2^{\lambda-1}}{\lambda} C_{\lambda} .
\end{aligned}
$$

Now let $0 \leq \varphi(x, t, r) \leq 1$. Then

$$
\begin{aligned}
A(x, t, r) & =C_{\lambda} \int_{\varphi(x, t, r)}^{1}(1-u)^{\lambda-1}(1+u)^{\lambda-1} d u=C_{\lambda} \int_{0}^{1-\varphi(x, t, r)} u^{\lambda-1}(2-u)^{\lambda-1} d u \\
& =C_{\lambda} \int_{\frac{1}{1-\varphi(x, t, r)}}^{\infty} u^{-\lambda-1}\left(2-\frac{1}{u}\right)^{\lambda-1} d u=C_{\lambda} \int_{\frac{1}{1-\varphi(x, t, r)}}^{\infty} u^{-2 \lambda}(2 u-1)^{\lambda-1} d u \\
& =2^{2 \lambda-1} C_{\lambda} \int_{\frac{2}{1-\varphi(x, t, r)}}^{\infty} u^{-2 \lambda}(u-1)^{\lambda-1} d u=2^{2 \lambda-1} C_{\lambda} \int_{\frac{1-\varphi(x, t, r)}{1+\varphi(x, t, r)}}^{\infty}(u+1)^{-2 \lambda} u^{\lambda-1} d u \\
& =2^{2 \lambda-1} C_{\lambda} \int_{0}^{\frac{1+\varphi(x, t) r}{1-\varphi(x, t, r)}}(1+u)^{-2 \lambda} u^{\lambda-1} d u \geq 2^{2 \lambda-1} C_{\lambda} \int_{0}^{1}(1+u)^{-2 \lambda} u^{\lambda-1} d u \\
& \geq 2^{2 \lambda-1} C_{\lambda} \int_{0}^{1} \frac{u^{\lambda-1}}{(1+u)^{2 \lambda}} \geq \frac{C_{\lambda}}{2} \int_{0}^{1} u^{\lambda-1} d u=\frac{C_{\lambda}}{2 \lambda} .
\end{aligned}
$$

Combining (2.7) and (2.8) for $-1 \leq \varphi(x, t, r) \leq 1$ we get

$$
A(x, t, r) \gtrsim 1 \text {. }
$$

From (2.6) and (2.9) we get (2.4) and consequently the assertion of Lemma 2.2. 
The following two inequalities are analogue of [13] and have an important role in proving our main results.

Lemma 2.3. Let $1<p \leq q<\infty$ and $v$ and $w$ be two functions measurable and positive a.e. on $(0, \infty)$. Then there exists a constant $C$ independent of the function $\varphi$ such that

$$
\left(\int_{0}^{\infty}\left(\int_{0}^{t} \varphi(\operatorname{ch} u) d u\right)^{q} w(\operatorname{ch} t) d t\right)^{\frac{1}{q}} \leq C\left(\int_{0}^{\infty} \varphi(\operatorname{ch} t)^{p} v(\operatorname{ch} t) d t\right)^{\frac{1}{p}}
$$

if and only if

$$
B=\sup _{r>0}\left(\int_{r}^{\infty} w(c h t) d t\right)^{\frac{1}{q}}\left(\int_{0}^{r} v(c h t)^{1-p^{\prime}} d t\right)^{\frac{1}{p^{\prime}}},
$$

where $p+p^{\prime}=p p^{\prime}$. Moreover, if $C$ is the best constant in (2.1), then

$$
B \leq C \leq k(p, q) B
$$

Here the constant $k(p, q)$ in (2.12) can be written in various forms. For example (see [7])

$$
k(p, q)=p^{\frac{1}{q}}\left(p^{\prime}\right)^{\frac{1}{p^{\prime}}} \text { or } k(p, q)=q^{\frac{1}{q}}\left(q^{\prime}\right)^{\frac{1}{p^{\prime}}} \text { or } k(p, q)=\left(1+\frac{q}{p^{\prime}}\right)^{\frac{1}{q}}\left(1+\frac{p^{\prime}}{q}\right)^{\frac{1}{p^{\prime}}} .
$$

Proof. Necessity. If $\varphi \geq 0$ and supp $\varphi \in[0, r]$, then

$$
\begin{aligned}
\int_{r}^{\infty}\left(\int_{0}^{t} \varphi(\operatorname{ch} u) d u\right)^{q} w(\operatorname{ch} t) d t & =\int_{r}^{\infty}\left[\left(\int_{0}^{r}+\int_{r}^{t}\right) \varphi(\operatorname{ch} u) d u\right]^{q} w(\operatorname{ch} t) d t \\
& =\int_{r}^{\infty}\left(\int_{0}^{r} \varphi(\operatorname{ch} u) d u\right)^{q} w(\operatorname{cht} t) d t
\end{aligned}
$$

For this we have

$$
\begin{aligned}
& \left(\int_{r}^{\infty}\left(\int_{0}^{t} \varphi(\operatorname{ch} u) d u\right)^{q} w(\operatorname{ch} t) d t\right)^{\frac{1}{q}}=\left(\int_{r}^{\infty} w(\operatorname{cht} t) d t\right)^{\frac{1}{q}}\left(\int_{0}^{r} \varphi(\operatorname{ch} u) d u\right) \\
\leq & \left(\int_{0}^{\infty}\left(\int_{0}^{t} \varphi(\operatorname{ch} u) d u\right)^{q} w(\operatorname{ch} t) d t\right)^{\frac{1}{q}} \leq C\left(\int_{0}^{\infty} \varphi(\operatorname{ch} t)^{p} v(\operatorname{ch} t) d t\right)^{\frac{1}{p}},
\end{aligned}
$$

i.e.,

$$
\left(\int_{r}^{\infty} w(\operatorname{ch} t) d t\right)^{\frac{1}{q}}\left(\int_{0}^{r} \varphi(\operatorname{ch} u) d u\right) \leq C\left(\int_{0}^{r} \varphi(\operatorname{ch} t)^{p} v(\operatorname{ch} t) d t\right)^{\frac{1}{p}} .
$$

Suppose

$$
\varphi(c h u)= \begin{cases}v(c h u)^{1-p^{\prime}} & \text { for } u \leq r \\ 0, & \text { for } u>r\end{cases}
$$


Then by (2.13)

$$
\begin{aligned}
& \left(\int_{r}^{\infty} w(\operatorname{ch} t) d t\right)^{\frac{1}{q}}\left(\int_{0}^{r} v(\operatorname{ch} u)^{1-p^{\prime}} d u\right) \\
\leq & C\left(\int_{0}^{r} v(\operatorname{ch} u)^{\left(1-p^{\prime}\right) p+1} d u\right)^{\frac{1}{p}}=C\left(\int_{0}^{r} v(\operatorname{ch} u)^{1-p^{\prime}} d u\right)^{\frac{1}{p}} .
\end{aligned}
$$

From this it follows that

$$
\left(\int_{r}^{\infty} w(\operatorname{ch} t) d t\right)^{\frac{1}{q}}\left(\int_{0}^{r} v(\operatorname{ch} u)^{1-p^{\prime}} d u\right)^{\frac{1}{p^{\prime}}} \leq C .
$$

Sufficiency. Suppose

$$
h(t)=\left(\int_{0}^{t} v(\operatorname{ch} u)^{1-p^{\prime}} d u\right)^{\frac{1}{q p^{\prime}}} .
$$

By Holder inequality we have

$$
\begin{aligned}
& \left(\int_{0}^{\infty}\left(\int_{0}^{t} \varphi(\operatorname{ch} u) d u\right)^{q} w(\operatorname{ch} t) d t\right)^{\frac{p}{q}} \\
= & \left(\int_{0}^{\infty}\left(\int_{0}^{t} \varphi(\operatorname{ch} u) h(u) v(\operatorname{ch} u)^{\frac{1}{p}} h^{-1}(u) v^{-\frac{1}{p}}(\operatorname{ch} u) d u\right)^{q} w(\operatorname{ch} t) d t\right)^{\frac{p}{q}} \\
\leq & \left\{\int_{0}^{\infty}\left(\int_{0}^{t} \varphi(\operatorname{ch} u) h(u) v(\operatorname{ch} u)^{\frac{1}{p}} d u\right)^{p} w(\operatorname{ch} t) d t\right. \\
& \left.\times\left(\int_{0}^{t} h(u)^{-p^{\prime}} v(\operatorname{ch} u)^{-\frac{p^{\prime}}{p}} d u\right)^{\frac{q}{p^{\prime}}} d t\right\}^{\frac{p}{q}} .
\end{aligned}
$$

Now we prove that if $\varphi, \psi \geq 0, r \geq 1$ then

$$
\left(\int_{0}^{\infty} \psi(\operatorname{ch} t)\left(\int_{0}^{t} \varphi(\operatorname{ch} u) d u\right)^{r} d t\right)^{\frac{1}{r}} \leq \int_{0}^{\infty} \varphi(\operatorname{ch} u)\left(\int_{u}^{\infty} \psi(\operatorname{ch} t) d t\right)^{\frac{1}{r}} d u .
$$

Indeed, since expression on the left hand in (2.17) is equal to

$$
\left(\int_{0}^{\infty}\left(\int_{0}^{\infty} \psi(\operatorname{ch} t)^{\frac{1}{r}} \varphi(\operatorname{ch} u) \chi_{[u, \infty)}(t) d u\right)^{r} d t\right)^{\frac{1}{r}},
$$

where $\chi_{[u, \infty)}$ is the characteristic function of the $[u, \infty)$, by Minkowsky inequality we have

$$
\int_{0}^{\infty}\left(\int_{0}^{\infty}\left(\psi(\operatorname{ch} t)^{\frac{1}{r}} \varphi(\operatorname{ch} u) \chi_{[u, \infty)}(t)\right)^{r} d t\right)^{\frac{1}{r}} d u \leq \int_{0}^{\infty} \varphi(\operatorname{ch} u)\left(\int_{u}^{\infty} \psi(\operatorname{ch} t) d t\right)^{\frac{1}{r}} d u .
$$


According to (2.17) right-hand (2.16) is estimate expression

$$
\int_{0}^{\infty}\left(\varphi(\operatorname{ch} u) h(u) v(\operatorname{ch} u)^{\frac{1}{p}}\right)^{p}\left(\int_{u}^{\infty}\left(\int_{0}^{t}\left(h(u)^{-p^{\prime}} v(\operatorname{ch} u)\right)^{\frac{-p^{\prime}}{p}} d u\right)^{\frac{q}{p^{\prime}}} w(\operatorname{ch} t) d t\right)^{\frac{p}{q}} d u .
$$

Take into account (2.15) in (2.18) we obtain

$$
\begin{aligned}
& \int_{u}^{\infty}\left(\int_{0}^{t}\left(h(u)^{-p^{\prime}} v(\operatorname{ch} u)\right)^{\frac{-p^{\prime}}{p}} d u\right)^{\frac{q}{p^{\prime}}} w(\operatorname{ch} t) d t \\
= & \int_{u}^{\infty}\left(\int_{0}^{t} v(\operatorname{ch} u)^{\frac{-p^{\prime}}{p}}\left(\int_{0}^{u} v(\operatorname{ch} x)^{1-p^{\prime}} d x\right)^{-\frac{1}{q}} d u\right)^{\frac{q}{p^{\prime}}} w(\operatorname{ch} t) d t .
\end{aligned}
$$

\section{Suppose}

$$
\psi(t)=\int_{0}^{t} v(\operatorname{ch} u)^{1-p^{\prime}} d u, \psi^{\prime}(t)=v(\operatorname{ch} t)^{1-p^{\prime}},
$$

we have

$$
\begin{aligned}
& \int_{0}^{t} v(\operatorname{ch} u)^{\frac{-p^{\prime}}{p}}\left(\int_{0}^{u} v(\operatorname{ch} x)^{1-p^{\prime}} d x\right) d u=\int_{0}^{t} v(\operatorname{ch} u)^{1-p^{\prime}} \psi(u)^{-\frac{1}{q}} d u \\
= & \int_{0}^{t} \psi^{\prime}(u) \psi(u)^{-\frac{1}{q}} d u=\int_{0}^{t} \psi(u)^{-\frac{1}{q}} d \psi(u)=\frac{q}{q-1} \psi(t)^{1-\frac{1}{q}} \\
= & q^{\prime}\left(\int_{0}^{t} v(\operatorname{ch} u)^{1-p^{\prime}} d u\right)^{\frac{1}{q^{\prime}}},
\end{aligned}
$$

then the integral (2.19) is equal to

$$
\left(q^{\prime}\right)^{\frac{q}{p^{\prime}}} \int_{u}^{\infty}\left(\int_{0}^{t} v(\operatorname{ch} u)^{1-p^{\prime}} d u\right)^{\frac{q}{p^{\prime} q^{\prime}}} w(\text { cht } t) d t
$$

From (2.11) it follows that

$$
\left(\int_{0}^{t} v(c h u)^{1-p^{\prime}} d u\right)^{\frac{1}{p^{\prime}}} \leq B\left(\int_{t}^{\infty} w(c h u) d u\right)^{-\frac{1}{q}}
$$

Therefore

$$
\left(\int_{0}^{t} v(\operatorname{chu})^{1-p^{\prime}} d u\right)^{\frac{q}{p^{\prime} q^{\prime}}} \leq B^{\frac{q}{q^{\prime}}}\left(\int_{t}^{\infty} w(\operatorname{ch} u) d u\right)^{-\frac{1}{q^{\prime}}} .
$$


From this and (2.20) it follows that

$$
\begin{aligned}
& \left(q^{\prime}\right)^{\frac{q}{p^{\prime}}} \int_{u}^{\infty}\left(\int_{0}^{t} v(\operatorname{ch} u)^{1-p^{\prime}} d u\right)^{\frac{q}{p^{\prime} q^{\prime}}} w(\text { cht }) d t \\
\leq & B^{\frac{q}{q^{\prime}}}\left(q^{\prime}\right)^{\frac{q}{p^{\prime}}} \int_{u}^{\infty} w(\operatorname{ch} t)\left(\int_{t}^{\infty} w(\operatorname{ch} t) d u\right)^{-\frac{1}{q^{\prime}}} d t \\
= & B^{q-1}\left(q^{\prime}\right)^{\frac{q}{p^{\prime}}} \int_{u}^{\infty}\left(\int_{t}^{\infty} w(\operatorname{ch} u) d u\right)^{\frac{1}{q}-1} w(\operatorname{ch} t) d t=M .
\end{aligned}
$$

Suppose

$$
\int_{u}^{\infty} w(\operatorname{cht}) d t=\mu(u) \Longrightarrow \mu^{\prime}(u)=w(\operatorname{ch} u)
$$

then

$$
\int_{u}^{\infty} \mu^{\prime}(t)(\mu(t))^{-\frac{1}{q^{\prime}}} d t=\int_{u}^{\infty} \mu(t)^{-\frac{1}{q^{\prime}}} d \mu(t)=\left.\frac{1}{1-\frac{1}{q^{\prime}}} \mu(t)^{1-\frac{1}{q^{\prime}}}\right|_{u} ^{\infty}=\frac{q^{\prime}}{q^{\prime}-1} \mu(u)^{\frac{1}{q}}=q \mu(u)^{\frac{1}{q}} .
$$

From this, (2.21), (2.11) and (2.15) we obtain

$$
\begin{aligned}
& M=B^{q-1}\left(q^{\prime}\right)^{\frac{q}{p^{\prime}}} q\left(\int_{u}^{\infty} w(\text { cht }) d t\right)^{\frac{1}{q}} \\
\leq & B^{q}\left(q^{\prime}\right)^{\frac{q}{p^{\prime}}} q\left(\int_{0}^{u} v(c h t)^{1-p^{\prime}} d t\right)^{-\frac{1}{p^{\prime}}}=B^{q}\left(q^{\prime}\right)^{\frac{q}{p^{\prime}}} q(h(u))^{-q} .
\end{aligned}
$$

Therefore the expression (2.18) is less than

$$
\begin{aligned}
& \int_{0}^{\infty}\left(\varphi(\operatorname{ch} u) h(u) v(\operatorname{ch} u)^{\frac{1}{p}}\right)^{p}\left(B^{q}\left(q^{\prime}\right)^{\frac{q}{p^{\prime}}} q(h(u))^{-q}\right)^{\frac{p}{q}} d u \\
= & B^{p}\left(q^{\prime}\right)^{\frac{p}{p^{\prime}}} q^{\frac{p}{q}} \int_{0}^{\infty} \varphi(\operatorname{ch} u)^{p} v(\operatorname{ch} u) d u .
\end{aligned}
$$

From this and (2.16) it follows that the inequality (2.10) holds with constant $B\left(q^{\prime}\right)^{\frac{1}{p^{\prime}}} q^{\frac{1}{q}}$. Moreover, if $C$ is the best constant in (2.1), then

$$
B \leq C \leq B\left(q^{\prime}\right)^{\frac{1}{p^{\prime}}} q^{\frac{1}{q}} .
$$

This completes the proof of the lemma.

Lemma 2.4. Let $1<p \leq q<\infty$ and let $v$ and $w$ be two functions measurable and positive a.e. on $(0, \infty)$. Then there exists a constant $C$ independent of the function $\varphi$ such that

$$
\left(\int_{0}^{\infty}\left(\int_{t}^{\infty} \varphi(\text { chu }) d u\right)^{q} w(\text { cht } t) d t\right)^{\frac{1}{q}} \leq C\left(\int_{0}^{\infty} \varphi(\text { cht })^{p} v(\text { ch } t) d t\right)^{\frac{1}{p}} .
$$


if and only if

$$
B_{1}=\sup _{r>0}\left(\int_{0}^{r} w(\operatorname{ch} t) d t\right)^{\frac{1}{q}}\left(\int_{r}^{\infty} v(\operatorname{ch} t)^{1-p^{\prime}} d t\right)^{\frac{1}{p^{\prime}}}<\infty .
$$

Moreover, the best constant $C$ in (2.22) satisfies the inequalities $B_{1} \leq C \leq k(p, q) B_{1}$.

Proof. Necessity. If $\varphi \geq 0$ and supp $\varphi \in[r, \infty)$, then

$$
\begin{aligned}
& \int_{0}^{r}\left(\int_{t}^{\infty} \varphi(\operatorname{ch} u) d u\right)^{q} w(\text { cht }) d t \\
= & \int_{0}^{r}\left[\left(\int_{t}^{r}+\int_{r}^{\infty}\right) \varphi(\operatorname{ch} u) d u\right]^{q} w(\operatorname{ch} t) d t=\int_{0}^{r}\left(\int_{r}^{\infty} \varphi(\operatorname{ch} u) d u\right)^{q} w(\operatorname{ch} t) d t .
\end{aligned}
$$

From this according to (2.22) we have

$$
\begin{aligned}
& \left(\int_{0}^{r}\left(\int_{r}^{\infty} \varphi(\operatorname{ch} u) d u\right)^{q} w(\operatorname{ch} t) d t\right)^{\frac{1}{q}}=\left(\int_{0}^{r} w(\operatorname{ch} t) d t\right)^{\frac{1}{q}} \int_{r}^{\infty} \varphi(\operatorname{ch} u) d u \\
\leq & \left(\int_{0}^{\infty}\left(\int_{r}^{\infty} \varphi(\operatorname{ch} u) d u\right)^{q} w(\text { cht }) d t\right)^{\frac{1}{q}} \leq C\left(\int_{0}^{\infty} \varphi(\operatorname{ch} t)^{\left.p_{v}(\text { ch } t) d t\right)^{\frac{1}{p}},}\right.
\end{aligned}
$$

i.e.

$$
\left(\int_{0}^{r} w(\operatorname{cht} t) d t\right)^{\frac{1}{q}} \int_{r}^{\infty} \varphi(\operatorname{ch} u) d u \leq C\left(\int_{r}^{\infty} \varphi(\operatorname{ch} t)^{p} v(\operatorname{ch} t) d t\right)^{\frac{1}{p}} .
$$

Suppose

$$
\varphi(\operatorname{ch} u)=\left\{\begin{array}{cc}
v(c h u)^{1-p^{\prime}}, & \text { for } u \geq r \\
0, & \text { for } u<r
\end{array}\right.
$$

Then by (2.24)

$$
\begin{aligned}
& \left(\int_{0}^{r} w(\operatorname{ch} t) d t\right)^{\frac{1}{q}}\left(\int_{r}^{\infty} v(\operatorname{chu})^{1-p^{\prime}} d u\right) \\
\leq & C\left(\int_{r}^{\infty} v(\operatorname{ch} u)^{\left(1-p^{\prime}\right) p+1} d u\right)^{\frac{1}{p}}=C\left(\int_{r}^{\infty} v(\operatorname{ch} u)^{1-p^{\prime}} d u\right)^{\frac{1}{p}} .
\end{aligned}
$$

From this it follows that

$$
\left(\int_{0}^{r} w(\operatorname{ch} t) d t\right)^{\frac{1}{q}}\left(\int_{r}^{\infty} v(\operatorname{ch} u)^{1-p^{\prime}} d u\right)^{\frac{1}{p^{\prime}}} \leq C .
$$

Sufficiency. Suppose

$$
h(t)=\left(\int_{t}^{\infty} v(\operatorname{chu})^{1-p^{\prime}} d u\right)^{\frac{1}{q p^{\prime}}}
$$


By Hölder inequality we have

$$
\begin{aligned}
& \left(\int_{0}^{\infty}\left(\int_{t}^{\infty} \varphi(\operatorname{chu}) d u\right)^{q} w(\operatorname{ch} t) d t\right)^{\frac{p}{q}} \\
= & \left(\int_{0}^{\infty}\left(\int_{t}^{\infty} \varphi(\operatorname{chu}) h(u) v(\operatorname{chu})^{\frac{1}{p}} h^{-1}(u) v^{-\frac{1}{p}}(\operatorname{ch} u) d u\right)^{q} w(\operatorname{ch} t) d t\right)^{\frac{p}{q}} \\
\leq & \left\{\int_{0}^{\infty}\left(\left(\int_{t}^{\infty} \varphi(\operatorname{ch} u) h(u) v(\operatorname{ch} u)^{\frac{1}{p}} d u\right)^{p} w(\operatorname{ch} t) d t\right)^{\frac{q}{p}}\right. \\
\times & \left.\left(\int_{t}^{\infty} h(u)^{-p^{\prime}} v(\operatorname{ch} u)^{-\frac{p^{\prime}}{p}} d u\right)^{\frac{q}{p^{\prime}}} d t\right\}^{\frac{p}{q}} .
\end{aligned}
$$

Now we prove that if $\varphi, \psi \geq 0, r \geq 1$ then

$$
\left(\int_{0}^{\infty} \psi(\operatorname{ch} t)\left(\int_{t}^{\infty} \varphi(\operatorname{ch} u) d u\right)^{r} d t\right)^{\frac{1}{r}} \leq \int_{0}^{\infty} \varphi(\operatorname{ch} u)\left(\int_{0}^{u} \psi(\operatorname{ch} t) d t\right)^{\frac{1}{r}} d u .
$$

Indeed, since expression on the left hand in (2.28) is equal to

$$
\left(\int_{0}^{\infty}\left(\int_{0}^{\infty} \psi(\operatorname{cht} t)^{\frac{1}{r}} \varphi(\operatorname{ch} u) \chi_{[0, u]}(t) d u\right)^{r} d t\right)^{\frac{1}{r}}
$$

where $\chi_{(0, u)}$-is the characteristic function on the $(0, u)$ and by Minkowsky inequality is less than

$$
\int_{0}^{\infty}\left(\int_{0}^{\infty}\left(\psi(\operatorname{ch} t)^{\frac{1}{r}} \varphi(\operatorname{ch} u) \chi_{(0, u)}(t)\right)^{r} d t\right)^{\frac{1}{r}} d u=\int_{0}^{\infty} \varphi(\operatorname{ch} u)\left(\int_{0}^{u} \psi(\operatorname{ch} t) d t\right)^{\frac{1}{r}} d u .
$$

According to (2.28) right-hand (2.27) is estimate by expression

$$
\int_{0}^{\infty}\left(\varphi(\operatorname{ch} u) h(u) v(\operatorname{ch} u)^{\frac{1}{p}}\right)^{p}\left(\int_{0}^{u}\left(\int_{t}^{\infty}\left(h(u)^{-p^{\prime}} v(\operatorname{ch} u)\right)^{-\frac{p^{\prime}}{p}} d u\right)^{\frac{q}{p^{\prime}}} w(\operatorname{ch} t) d t\right)^{\frac{p}{q}} d u .
$$

Take into account (2.26) in (2.29) we obtain

$$
\int_{0}^{u}\left(\int_{t}^{\infty} v(\operatorname{ch} u)^{1-p^{\prime}}\left(\int_{u}^{\infty} v(\operatorname{ch} x)^{1-p^{\prime}} d x\right)^{-\frac{1}{q}} d u\right)^{\frac{q}{p^{\prime}}} w(\operatorname{ch} t) d t
$$

Suppose

$$
\psi(t)=\int_{t}^{\infty} v(\operatorname{ch} u)^{1-p^{\prime}} d u, \quad \psi^{\prime}(t)=v(\operatorname{ch} t)^{1-p^{\prime}}
$$


we have

$$
\begin{aligned}
& \int_{t}^{\infty} v(\operatorname{ch} u)^{1-p^{\prime}}\left(\int_{u}^{\infty} v(\operatorname{ch} x)^{1-p^{\prime}} d x\right) d u=\int_{t}^{\infty} v(\operatorname{ch} u)^{1-p^{\prime}} \psi(u)^{-\frac{1}{q}} d u \\
= & \int_{t}^{\infty} \psi^{\prime}(u) \psi(u)^{-\frac{1}{q}} d u=\int_{t}^{\infty} \psi(u)^{-\frac{1}{q}} d \psi(u) \\
= & \frac{q}{q^{\prime}} \psi(t)^{1-\frac{1}{q}}=q^{\prime}\left(\int_{t}^{\infty} v(\operatorname{ch} u)^{1-p^{\prime}} d u\right)^{\frac{1}{q^{\prime}}},
\end{aligned}
$$

but then the integral (2.30) is equal to

$$
\left(q^{\prime}\right)^{\frac{q}{p^{\prime}}} \int_{0}^{u}\left(\int_{t}^{\infty} v(\operatorname{ch} u)^{1-p^{\prime}} d u\right)^{\frac{q}{p^{\prime} q^{\prime}}} w(\text { cht } t) d t .
$$

From (2.23) it follows that

$$
\left(\int_{t}^{\infty} v(\operatorname{ch} u)^{1-p^{\prime}} d u\right)^{\frac{1}{p^{\prime}}} \leq B_{1}\left(\int_{0}^{t} w(\operatorname{ch} u) d u\right)^{-\frac{1}{q}},
$$

but then

$$
\left(\int_{t}^{\infty} v(\operatorname{ch} u)^{1-p^{\prime}} d u\right)^{\frac{q}{p^{\prime} q^{\prime}}} \leq B_{1}^{\frac{q}{q^{\prime}}}\left(\int_{0}^{t} w(\operatorname{ch} u) d u\right)^{-\frac{1}{q^{\prime}}} .
$$

From this and (2.31) it follows that

$$
\begin{aligned}
& \left(q^{\prime}\right)^{\frac{q}{p^{\prime}}} \int_{0}^{u}\left(\int_{t}^{\infty} v(\operatorname{ch} u)^{1-p^{\prime}} d u\right)^{\frac{q}{p^{\prime} q^{\prime}}} w(\text { cht }) d t \\
\leq & B_{1}^{\frac{q}{q^{\prime}}}\left(q^{\prime}\right)^{\frac{q}{p^{\prime}}} \int_{0}^{u} w(\text { cht } t)\left(\int_{0}^{t} w(\operatorname{ch} u) d u\right)^{-\frac{1}{q^{\prime}}} d t \\
= & B_{1}^{q-1}\left(q^{\prime}\right)^{\frac{q}{p^{\prime}}} \int_{0}^{u}\left(\int_{0}^{t} w(\operatorname{ch} u) d u\right)^{\frac{1}{q}-1} w(\operatorname{ch} t) d t=M_{1} .
\end{aligned}
$$

Suppose

$$
\int_{0}^{u} w(\text { cht }) d t=\theta(u) \Rightarrow \theta^{\prime}(u)=w(\operatorname{chu})
$$

then

$$
\int_{0}^{u} \theta^{\prime}(t) \theta(t)^{-\frac{1}{q^{\prime}}} d t=\int_{0}^{u} \theta(t)^{-\frac{1}{q^{\prime}}} d \theta(t)=q \theta(u)^{\frac{1}{q}} .
$$

From this, (2.32), (2.23) and (2.26) we obtain

$$
\begin{aligned}
& M_{1}=B_{1}^{q-1}\left(q^{\prime}\right)^{\frac{q}{p^{\prime}}} q\left(\int_{0}^{u} w(\text { cht } t) d t\right)^{\frac{1}{q}} \\
\leq & B_{1}^{q}\left(q^{\prime}\right)^{\frac{q}{p^{\prime}}} q\left(\int_{u}^{\infty} w(\text { cht } t)^{1-p^{\prime}} d t\right)^{-\frac{1}{p^{\prime}}}=B_{1}^{q}\left(q^{\prime}\right)^{\frac{q}{p^{\prime}}} q(h(u))^{-q} .
\end{aligned}
$$


Therefore the expression (2.29) is less than

$$
\begin{aligned}
& \int_{0}^{\infty}\left(\varphi(\operatorname{chu}) h(u) v(\operatorname{chu})^{\frac{1}{p}}\right)^{p}\left(B_{1}^{q}\left(q^{\prime}\right)^{\frac{q}{p^{\prime}}}(h(u))^{-q}\right)^{\frac{p}{q}} d u \\
= & B_{1}^{p}\left(q^{\prime}\right)^{\frac{p}{p^{\prime}}} q^{\frac{p}{q}} \int_{0}^{\infty} \varphi(\operatorname{chu})^{p} v(\text { chu }) d u .
\end{aligned}
$$

From this and (2.27) it follows that the inequality (2.22) holds with constant $B_{1}\left(q^{\prime}\right)^{\frac{1}{p^{\prime}}} q^{\frac{1}{q}}$.

\section{$3 \mathrm{O}^{\prime}$ Neil inequality for rearrangements of G-convolution}

In this section, we will establish a relation between shift operator $A_{\text {cht }}^{\lambda}$ and $\lambda$-rearrangement of $f$. We show that for the G-convolution an $\mathrm{O}^{\prime} \mathrm{Neil}$ inequality for rearrangements holds. Let $f: \mathbb{R}_{+} \rightarrow \mathbb{R}$ be a measurable function and for any measurable set $E, \mu E=|E|_{\lambda}=\int_{E} s h^{2 \lambda} x d x$. We define $\lambda$-rearrangement of $f$ in decreasing order by

$$
f^{*}(\operatorname{cht})=\inf \left\{u>0: f_{*}(u) \leq \operatorname{sh} \frac{t}{2}\right\}, \quad t>0,
$$

where $f_{*}$ denotes the $\lambda$-distribution function of $f$ given by

$$
f_{*}(u)=\left|\left\{x \in \mathbb{R}_{+}:|f(\operatorname{ch} x)|>u\right\}\right|_{\lambda}, \quad u \geq 0 .
$$

Further we need some properties of $\lambda$-rearrangement of functions which are analogous from $[1,7]$.

Observe that $f_{*}$ depends only on the absolute value $|f|$ of the function $f$, and $f_{*}$ may assume the value $+\infty$.

Proposition 3.1. Let $f, g, f_{n},(n=1,2, \ldots)$ measurable and nonnegative functions on $\mathbb{R}_{+}$. Then

(i) $f_{*}$ is decreasing and right-continuous on $[0, \infty)$.

(ii) If $|f(\operatorname{ch} x)| \leq|g(\operatorname{ch} x)| \mu-$ a.e., then $f_{*}(u) \leq g_{*}(u)$ for $u \geq 0$.

(iii) If $|f(\operatorname{ch} x)| \leq \liminf _{n \rightarrow \infty}\left|f_{n}(\operatorname{ch} x)\right| \mu$-a.e., then

$$
f_{*}(u) \leq \liminf _{n \rightarrow \infty}\left(f_{n}\right)_{*}(u) \text { for } u \geq 0 .
$$

The proof of this properties is precisely the same how the Proposition 1.7 from [7].

Proposition 3.2. The following equality is valid

$$
f^{*}(\operatorname{ch} t)=m_{f_{*}}\left(\operatorname{sh} \frac{t}{2}\right), t \geq 0
$$

where $m$ is the Lebesgue measure. 
Proof. Since $f_{*}$ is a decreasing function by Proposition 3.1 (i) it follows that

$$
\sup \left\{u: f_{*}(u)>\operatorname{sh} \frac{t}{2}\right\}=m\left\{u: f_{*}(u)>\operatorname{sh} \frac{t}{2}\right\} \text {. }
$$

Hence we get

$$
\begin{aligned}
f^{*}(\operatorname{ch} t) & =\inf \left\{u: f_{*}(u) \leq \operatorname{sh} \frac{t}{2}\right\}=\sup \left\{u: f_{*}(u)>\operatorname{sh} \frac{t}{2}\right\} \\
& =m\left\{u: f_{*}(u)>\operatorname{sh} \frac{t}{2}\right\}=m_{f_{*}}\left(\operatorname{sh} \frac{t}{2}\right) .
\end{aligned}
$$

The next proposition establish some properties of the decreasing rearrangement.

Proposition 3.3. The following properties holds.

(i) $f^{*}($ cht $)>u \Longleftrightarrow f_{*}(u)>\operatorname{sh} \frac{t}{2}$;

(ii) $f$ and $f^{*}$ are equimeasurable, that is, for all

$$
f_{*}(u)=\left|\left\{x \in \mathbb{R}_{+}:|f(\operatorname{ch} x)|>u\right\}\right|_{\lambda}=m\left(\operatorname{sh} \frac{t}{2}>0: f^{*}(\operatorname{ch} t)>u\right)=m_{f^{*}}(u) ;
$$

(iii) If $E \in \mathbb{R}_{+}$, then

$$
\left(f \chi_{E}\right)^{*}(\operatorname{ch} t) \leq f^{*}(\operatorname{ch} t) \chi_{\left[0,|E|_{\lambda}\right]}(\operatorname{ch} t), t>0 ;
$$

(iv) If $u \geq 0$ and $f_{*}(u)<\infty$, then

$$
f^{*}\left(f_{*}(u)\right) \leq u \text { and } f^{*}\left(f_{*}(u)-\varepsilon\right) \geq u \text { for all } 0<\varepsilon<f^{*}(\text { cht }) .
$$

If $t \geq 0$ and $f^{*}($ cht $)<\infty$, then

$$
f_{*}\left(f^{*}(\operatorname{ch} t)\right) \leq \operatorname{sh} \frac{t}{2} \text { and } f_{*}\left(f^{*}(\text { cht })-\varepsilon\right) \geq \operatorname{sh} \frac{t}{2} \text { for all } \varepsilon>0 .
$$

Proof. (i) First assume that $f_{*}(u)>\operatorname{sh} \frac{t}{2}$. Then, since $f_{*}$ is a decreasing function, we have

$$
\inf \left\{v: f_{*}(v) \leq \operatorname{sh} \frac{t}{2}\right\}>u
$$

Thus $f^{*}($ cht $)>u$.

Now assume that

$$
f^{*}(\operatorname{ch} t)>u \Longleftrightarrow \inf \left\{v: f_{*}(v) \leq \operatorname{sh} \frac{t}{2}\right\}>u
$$

Thus, since $f_{*}$ is a decreasing function, we get $f_{*}(u)>\operatorname{sh} \frac{t}{2}$. 
(ii) Let $m$ be the Lebesgue measure on $\mathbb{R}_{+}$. Then by (i) we get

$$
\begin{aligned}
m_{f^{*}}(u) & =m\left(\operatorname{sh} \frac{t}{2} \geq 0: f^{*}(\operatorname{ch} t)>u\right)=m\left(\operatorname{sh} \frac{t}{2} \geq 0: f_{*}(u)>\operatorname{sh} \frac{t}{2}\right) \\
& =m\left[0, f_{*}(u)\right]=f_{*}(u)=\left|\left\{x \in \mathbb{R}_{+}:|f(\operatorname{ch} x)|>u\right\}\right|_{\lambda} .
\end{aligned}
$$

(iii) Since $\left(f \chi_{E}\right)(\operatorname{ch} x) \leq f(\operatorname{ch} x)$ for all $x \in E$ we have by Proposition 3.1 (ii) and Proposition 3.2 that

$$
\begin{aligned}
&\left(f \chi_{E}\right)_{*}(u) \leq f_{*}(u), u \geq 0 \Longleftrightarrow \inf \left\{u \geq 0:\left(f \chi_{E}\right)_{*}(u) \leq \operatorname{sh} \frac{t}{2}\right\} \\
& \Longleftrightarrow \inf \left\{u \geq 0: f_{*}(u) \leq \operatorname{sh} \frac{t}{2}\right\} \Longleftrightarrow\left(f \chi_{E}\right)^{*}(\text { cht }) \leq f^{*}(\text { cht }), \quad t \geq 0 .
\end{aligned}
$$

On the other hand, since

$$
\left(f \chi_{E}\right)_{*}(u)=\left|\left\{x \in \mathbb{R}_{+}:\left|\left(f \chi_{E}\right)(\operatorname{ch} x)\right|>u\right\}\right|_{\lambda} \leq|E|_{\lambda}
$$

we have

$$
\left(f \chi_{E}\right)^{*}(\operatorname{ch} t)=0, \quad \text { cht }>|E|_{\lambda} .
$$

Combining these two estimates we can conclude that

$$
\left(f \chi_{E}\right)^{*}(\operatorname{ch} t) \leq f^{*}(\operatorname{ch} t) \chi_{\left[0,|E|_{\lambda}\right]}(\operatorname{ch} t), t>0 .
$$

(iv) Assume that $f_{*}(u)<\infty$. Since $f_{*}$ is a decreasing function then suppose by assuming that $c h t=f_{*}(u)$ we get

$$
\begin{aligned}
f^{*}\left(f_{*}(u)\right) & =f^{*}(\operatorname{cht})=\inf \left\{s \geq 0: f_{*}(s) \leq \operatorname{sh} \frac{t}{2}\right\} \\
& \leq \inf \left\{s \geq 0: f_{*}(s)<\operatorname{cht}=f_{*}(u)\right\} \leq u .
\end{aligned}
$$

Also, for all $\varepsilon>0$

$$
f^{*}\left(f_{*}(u)-\varepsilon\right)=\inf \left\{s \geq 0: f_{*}(s) \leq f_{*}(u)-\varepsilon\right\} \geq u .
$$

Now assume that $f^{*}(\operatorname{cht} t)<\infty$, then

$$
f_{*}\left(f^{*}(\operatorname{cht})\right)=f_{*}\left(\inf \left\{u \geq 0: f_{*}(u) \leq \operatorname{sh} \frac{t}{2}\right\}\right) \leq \operatorname{sh} \frac{t}{2}
$$

by the right-continuity of $f_{*}$. Furthermore, for all $\varepsilon>0$ by (ii) we have

$$
\begin{aligned}
& f_{*}\left(f^{*}(\operatorname{ch} t)-\varepsilon\right)=\left|\left\{x \in \mathbb{R}_{+}:|f(\operatorname{ch} x)|>f^{*}(\operatorname{ch} t)-\varepsilon\right\}\right|_{\lambda} \\
= & m\left(\left\{s>0: f^{*}(\text { chs })>f^{*}(\text { ch } t)-\varepsilon\right\}\right) \geq \operatorname{sh} \frac{t}{2} .
\end{aligned}
$$

This completes the proof of the proposition. 
Proposition 3.4. For any $E \subset \mathbb{R}$ the following equalities are valid

$$
\int_{E}|f(\operatorname{ch} x)| \operatorname{sh}^{2 \lambda} x d x=\int_{0}^{\infty} f_{*}(u) d u=\int_{0}^{\infty} f^{*}(\operatorname{cht} t) d t .
$$

Proof. We first prove (3.1) for simple positive functions. Let $f$ be a positive simple function on $E$ of the form

$$
f(\operatorname{ch} x)=\sum_{j=1}^{n} \alpha_{j} \chi_{E_{j}}(\operatorname{ch} x),
$$

where $\alpha_{1}>\alpha_{2}>\ldots>\alpha_{n}>0, E_{j}=\left\{x \in \mathbb{R}_{+}: f(\operatorname{ch} x)=\alpha_{j}\right\}$ and $\chi_{E}$ is the characteristic function of the set $E$. All $E_{j}$ are pairwise disjoint sets. Then

$$
f_{*}(u)=\sum_{j=1}^{n} \beta_{j} \chi_{B_{j}}(u),
$$

where $\beta_{j}=\sum_{i=1}^{j}\left|E_{i}\right|_{\lambda}, B_{n}=\left[\alpha_{j+1}, \alpha_{j}\right), j=1, \ldots, n$ and $\alpha_{n+1}=0$.

Thus we have

$$
\begin{aligned}
& \int_{0}^{\infty} f_{*}(u) d u=\int_{0}^{\infty}\left(\sum_{j=1}^{n} \beta_{j} \chi_{\left[\alpha_{j+1}, a_{j}\right)}(u)\right) d u \\
= & \sum_{j=1}^{n} \beta_{j} \int_{\alpha_{j+1}}^{\alpha_{i}} d u=\sum_{j=1}^{n} \beta_{j}\left(\alpha_{j}-\alpha_{j+1}\right) \\
= & \beta_{1}\left(\alpha_{1}-\alpha_{2}\right)+\beta_{2}\left(\alpha_{2}-\alpha_{3}\right)+\ldots+\beta_{n} \alpha_{n} \\
= & \alpha_{1} \beta_{1}-\alpha_{2} \beta_{2}+\alpha_{2} \beta_{2}-\alpha_{3} \beta_{3}+\ldots+\alpha_{n-1} \beta_{n-1}-\alpha_{n} \beta_{n-1}+\alpha_{n} \beta_{n} \\
= & \alpha_{1} \beta_{1}+\alpha_{2}\left(\beta_{2}-\beta_{1}\right)+\ldots+\alpha_{n-1}\left(\beta_{n-1}-\beta_{n-2}\right)+\alpha_{n} \beta_{n}=\sum_{j=1}^{n} \alpha_{j}\left|E_{j}\right|_{\lambda} .
\end{aligned}
$$

Further since

$$
f^{*}(\text { cht })=\sum_{j=1}^{n} \alpha_{j} \chi_{\left[\beta_{j-1}, \beta_{j}\right)}(\text { cht }),
$$

then

$$
\begin{aligned}
& \int_{0}^{\infty} f^{*}(\text { cht }) d t \\
= & \int_{0}^{\infty}\left(\sum_{j=1}^{n} \alpha_{j} \chi_{\left[\beta_{j-1}, \beta_{j}\right)}(\operatorname{ch} x)\right) d t \\
= & \sum_{j=1}^{n} \alpha_{j} \int_{\beta_{j-1}}^{\beta_{i}} d t=\sum_{j=1}^{n} \alpha_{j}\left(\beta_{j}-\beta_{j-1}\right)=\sum_{j=1}^{n} \alpha_{j}\left|E_{j}\right|_{\lambda} .
\end{aligned}
$$


As $E=\sum_{j=1}^{n} E_{j}$, then

$$
\sum_{j=1}^{n} \alpha_{j}\left|E_{j}\right|_{\lambda}=\sum_{j=1}^{n} \int_{E_{j}} f(\operatorname{ch} x) \operatorname{sh}^{2 \lambda} x d x=\int_{E} f(\operatorname{ch} x) \operatorname{sh}^{2 \lambda} x d x .
$$

From this, (3.1) and (3.4) it follows that (3.1) is satisfied for simple functions. The general case follows from Proposition 3.1 (iii), Proposition 3.2 and the monotone convergence theorem.

Proposition 3.5. Let $0<p<\infty$. Then

$$
\int_{0}^{\infty} \mid\left. f(\text { cht } t)\right|^{p} s h^{2 \lambda} t d t=p \int_{0}^{\infty} u^{p-1} f_{*}(u) d u=\int_{0}^{\infty} f^{*}(\text { cht })^{p} d t .
$$

Proof. Since $f$ is $\mu$-measurable function, $\|f\|^{p}$ is a $\mu$-measurable function for $0<p<\infty$. By Proposition 3.3 (ii) it follows that $|f|^{p}$ and $\left(f^{*}\right)^{p}$ is equimeasurable, then by Proposition 3.4 we have

$$
\begin{aligned}
& \int_{0}^{\infty}|f(c h t)|^{p} \operatorname{sh}^{2 \lambda} t d t=p \int_{0}^{\infty}\left(\int_{0}^{|f(c h t)|} u^{p-1} d u\right) s h^{2 \lambda} t d t \\
= & p \int_{0}^{\infty} u^{p-1}\left(\int_{\{t \in[0, \infty):|f(c h t)>u|\}} s h^{2 \lambda} t d t\right) d u \\
= & p \int_{0}^{\infty} u^{p-1}|\{t \in[0, \infty):|f(c h t)|>u\}|_{\lambda} d u \\
= & p \int_{0}^{\infty} u^{p-1} f_{*}(u) d u=\int_{0}^{\infty} f^{*}(\text { cht })^{p} d t .
\end{aligned}
$$

This completes the proof of the proposition.

Proposition 3.6. For any measurable $E \subset \mathbb{R}_{+}$such that $|E|_{\lambda} \leq t$ the following inequalities are valid

$$
\int_{E}|f(\operatorname{ch} x)| \operatorname{sh}^{2 \lambda} x d x \leq \int_{0}^{|E|_{\lambda}} f^{*}(\operatorname{ch} u) d u \leq \int_{0}^{t} f^{*}(\operatorname{ch} u) d u .
$$

Proof. If $t=\infty$, then the inequality is true by Proposition 3.4. Assume that $t<\infty$. Then by Proposition 3.4 and Proposition 3.3 (iii) we obtain

$$
\begin{aligned}
& \int_{E}|f(\operatorname{ch} x)| \operatorname{sh}^{2 \lambda} x d x=\int_{0}^{\infty}\left|f(\operatorname{ch} x) \chi_{E}(\operatorname{ch} x)\right| \operatorname{sh}^{2 \lambda} x d x=\int_{0}^{\infty}\left(f \chi_{E}\right)^{*}(\operatorname{ch} u) d u \\
\leq & \int_{0}^{\infty} f^{*}(\operatorname{ch} u) \chi_{\left[0,|E|_{\lambda}\right]}(\operatorname{ch} u) d u=\int_{0}^{|E|_{\lambda}} f^{*}(\operatorname{ch} u) d u \leq \int_{0}^{t} f^{*}(\operatorname{ch} u) d u .
\end{aligned}
$$

From Proposition 3.6 we immediately obtain the inequality

$$
\sup _{\sup |E|_{\lambda}=t} \int_{E}|f(\operatorname{ch} x)| \operatorname{sh}^{2 \lambda} x d x \leq \int_{0}^{t} f^{*}(\operatorname{ch} u) d u \text {. }
$$


Proposition 3.7. Let $f$ and $g$ be measurable functions on $\mathbb{R}_{+}$. Then the following inequality is valid

$$
\int_{0}^{\infty}|f(\operatorname{ch} x) g(\operatorname{ch} x)| \operatorname{sh}^{2 \lambda} x d x \leq \int_{0}^{\infty} f^{*}(\operatorname{ch} t) g^{*}(\operatorname{ch} t) d t .
$$

Proof. We prove this inequality for positive simple functions and the general results will follow by Proposition 3.1(iii), Proposition 3.2 and the monotone convergence theorem for measurable functions on $\mathbb{R}_{+}$. Let $f$ be a simple function of the form

$$
f(\operatorname{ch} x)=\sum_{j=1}^{n} \alpha_{j} \chi_{E_{j}}(\operatorname{ch} x),
$$

where $\alpha_{1}>\alpha_{2}>\ldots>\alpha_{n}$ and $E_{j} \subset \mathbb{R}_{+}, E_{j} \cap E_{k}=\varnothing, k \neq j$. Let

$$
B_{j}=\sum_{i=1}^{j} E_{i}, \quad \gamma_{j}=\sum_{i=1}^{j}\left|E_{i}\right|_{\lambda}, \quad \gamma_{0}=0
$$

and $\beta_{j}=\alpha_{j}-\alpha_{j+1}, \alpha_{n+1}=0$. By Proposition 3.6 we get

$$
\begin{aligned}
& \int_{0}^{\infty}|f(\operatorname{ch} x) g(\operatorname{ch} x)| \operatorname{sh}^{2 \lambda} x d x=\int_{0}^{\infty}\left|\left(\sum_{j=1}^{n} \beta_{j} \chi_{B_{j}}(\operatorname{ch} x)\right) g(\operatorname{ch} x)\right| \operatorname{sh}^{2 \lambda} x d x \\
& =\sum_{j=1}^{n} \beta_{j} \int_{B_{j}}|g(\operatorname{ch} x)| s h^{2 \lambda} x d x \leq \sum_{j=1}^{n} \beta_{j} \int_{0}^{\left|B_{j}\right| \lambda} g^{*}(\operatorname{ch} t) d t \\
& =\sum_{j=1}^{n}\left(\alpha_{j}-\alpha_{j+1}\right) \int_{0}^{\left|B_{j}\right|_{\lambda}} g^{*}(\text { cht } t) d t=\sum_{j=1}^{n} \alpha_{j} \int_{\gamma_{j-1}}^{\gamma_{j}} g^{*}(\text { ch } t) d t \\
& =\int_{0}^{\infty}\left(\sum_{j=1}^{n} \alpha_{j} \chi_{\left[\gamma_{j-1}, \gamma_{j}\right)}(\text { cht })\right) g^{*}(\text { cht }) d t=\int_{0}^{\infty} f^{*}(\text { cht }) g^{*}(\text { cht }) d t .
\end{aligned}
$$

Proposition 3.8. For any $t>0$ the following equality is valid

$$
\sup _{|E|_{\lambda}=t} \int_{E}|f(\operatorname{ch} x)| \operatorname{sh}^{2 \lambda} x d x=\int_{0}^{t} f^{*}(\operatorname{ch} u) d u \text {. }
$$

Proof. We need to consider two separate cases, according to whether a number does or does not lie in the range of the distribution function $f_{*}$ of $f$. Suppose first there exists a $v>0$ for which $f_{*}(v)=\operatorname{sh} \frac{a}{2}$. In that case, it follows that (see Proposition 3.3 (iv))

$$
f^{*}\left(f_{*}(v)\right)=f^{*}\left(\operatorname{sh} \frac{a}{2}\right) \leq v
$$

and so

$$
\operatorname{sh} \frac{a}{2}=f_{*}(v) \leq f_{*}\left(f^{*}\left(\operatorname{sh} \frac{a}{2}\right)\right) \leq f_{*}\left(f^{*}(\operatorname{ch} a)\right) \leq \operatorname{sh} \frac{a}{2}
$$


i.e.,

$$
f_{*}\left(f^{*}\left(\operatorname{sh} \frac{a}{2}\right)\right)=\operatorname{sh} \frac{a}{2}
$$

Therefore, let

$$
E=\left\{x \in \mathbb{R}_{+}:|f(\operatorname{ch} x)|>f^{*}\left(\operatorname{sh} \frac{a}{2}\right)\right\}
$$

Then

$$
f_{*}\left(\chi_{E}(u)\right)=f_{*}\left(\max \left\{u, f^{*}\left(\operatorname{sh} \frac{a}{2}\right)\right\}\right)
$$

and by the equimeasurability of $f$ and $f^{*}$ (see Proposition 3.3(ii)) we have

$$
\begin{aligned}
& m_{f^{*} \chi\left[0, s h \frac{a}{2}\right]}(u) \leq \min \left\{m_{f^{*}}(u), \operatorname{sh} \frac{a}{2}\right\}=\min \left\{f_{*}(u), \operatorname{sh} \frac{a}{2}\right\} \\
= & \min \left\{f^{*}(u), f_{*}\left(f^{*}\left(\operatorname{sh} \frac{a}{2}\right)\right)\right\}=f_{*}\left(\max \left\{u, f^{*}\left(\operatorname{sh} \frac{a}{2}\right)\right\}\right) .
\end{aligned}
$$

Let $\varepsilon>0$ be arbitrary and take $c h t_{0}=\min \left\{f_{*}(u), \operatorname{sh} \frac{a}{2}\right\}-\varepsilon$. Then by Proposition 3.3(iv)

$$
f^{*}\left(\operatorname{cht}_{0}\right) \chi_{\left[0, s h \frac{a}{2}\right]}\left(\operatorname{cht}_{0}\right)=f^{*}\left(\operatorname{cht} t_{0}\right) \geq f^{*}\left(f_{*}(u)-\varepsilon\right) \geq u,
$$

that is

$$
\begin{aligned}
m_{f^{*} \chi_{\left[0, s h \frac{a}{2}\right]}}(u) & =m\left\{t>0: f^{*}(\operatorname{cht} t) \chi_{\left[0, s h \frac{a}{2}\right]}(\operatorname{cht} t)>u\right\} \\
\geq & \operatorname{cht}_{0}=\min \left\{f_{*}(u), \operatorname{sh} \frac{a}{2}\right\}-\varepsilon .
\end{aligned}
$$

From (3.6) and (3.7) since $\varepsilon$ was arbitrary, we have

$$
m_{\left.f^{*} \chi_{[0, s h}\right]}(u)=f_{*}\left(\max \left\{u, f^{*}\left(\operatorname{sh} \frac{a}{2}\right)\right\}\right)=f_{*}\left(\chi_{E}(u)\right)
$$

for $u \geq 0$. Hence $f \chi_{E}$ and $f^{*} \chi_{\left[0, s h \frac{a}{2}\right]}$ are equimeasurable and because by (3.1) we obtain

$$
\begin{aligned}
& \int_{E}|f(\operatorname{ch} x)| \operatorname{sh}^{2 \lambda} x d x=\int_{0}^{\infty}\left|f \chi_{E}(\operatorname{ch} x)\right| \operatorname{sh}^{2 \lambda} x d x \\
= & \int_{0}^{\infty} f^{*}(\operatorname{ch} t) \chi_{\left[0, s h \frac{a}{2}\right]}(\operatorname{cht} t) d t=\int_{0}^{\operatorname{sh} \frac{a}{2}} f^{*}(\operatorname{ch} u) d u .
\end{aligned}
$$

Take $s h \frac{a}{2}=t$ we obtain (3.5). The case where $t$ is not the range of $f_{*}$ prove the same when of Lemma 2.5 from [7].

The function $f^{* *}$ on $\mathbb{R}_{+}$is defined by

$$
f^{* *}(\operatorname{ch} t)=\frac{1}{t} \int_{0}^{t} f^{*}(\operatorname{ch} u) d u, t>0 .
$$


Since $f^{*}$ is decreasing then

$$
f^{* *}(\operatorname{ch} t)=\frac{1}{t} \int_{0}^{t} f^{*}(\operatorname{ch} u) d u \geq f^{*}(\operatorname{ch} t) \cdot \frac{1}{t} \int_{0}^{t} d u=f^{*}(\operatorname{ch} t) .
$$

We denote by $W L_{p, \lambda}\left(\mathbb{R}_{+}\right)$the weak $L_{p, \lambda}$ space of all measurable functions $f$ with finite norm

$$
\|f\|_{W L_{p, \lambda}}=\sup _{t>0}\left(\operatorname{sh} \frac{t}{2}\right)^{\frac{1}{p}} f^{*}(\text { cht }), \quad 1 \leq p<\infty .
$$

Lemma 3.1. For any measurable set $E \subset \mathbb{R}_{+}$and for any $y \in \mathbb{R}_{+}$

$$
\sup _{|E|_{\lambda}=t} \int_{E} A_{c h y}^{\lambda}|f(\operatorname{ch} x)| \operatorname{sh}^{2 \lambda} x d x \approx \int_{0}^{t} f^{*}(\operatorname{ch} u) d u .
$$

These inequalities immediate follows from Lemma 2.2 and (2.28).

The following theorem is one of our main results which shows that an $\mathrm{O}^{\prime}$ Neil inequality for rearrangements of the G-convolution holds. The methods of the proof used here are close to those [6].

Theorem 3.1. Let $f, g$ be positive measurable functions on $\mathbb{R}_{+}$. Then for all $0<t<\infty$

$$
(f \oplus g)^{* *}(\operatorname{ch} t) \lesssim f^{* *}(\operatorname{ch} t) \int_{0}^{t} g^{* *}(\operatorname{ch} u) d u+\int_{t}^{\infty} f^{*}(\operatorname{ch} u) g^{* *}(\operatorname{ch} u) d u .
$$

Proof. For $t>0$ we choose a measurable set $E_{t}$ such that

$$
\left\{x \in \mathbb{R}_{+}: \mid f(\text { cht }) \mid>f^{*}(\text { cht } t)\right\} \subset E_{t} \subset\left\{x \in \mathbb{R}_{+}:|f(\operatorname{ch} x)| \geq f^{*}(\text { cht })\right\} .
$$

Let

$$
f_{1}(\operatorname{ch} x)=\left(f(\operatorname{ch} x)-f^{*}(\operatorname{ch} t)\right) \chi_{E_{t}}(x), f_{2}(\operatorname{ch} x)=f(\operatorname{ch} x)-f_{1}(\operatorname{ch} x) .
$$

For any measurable set $\mathbb{A} \subset \mathbb{R}_{+}$with measure $|\mathbb{A}|_{\lambda}=t$, we have

$$
\begin{aligned}
\int_{\mathbb{A}}\left(g \oplus f_{1}\right)(\operatorname{ch} x) \operatorname{sh}^{2 \lambda} x d x & =\int_{\mathbb{A}}\left(\int_{\mathbb{R}_{+}} f_{1}(\operatorname{ch} y) A_{c h y}^{\lambda} g(\operatorname{ch} x) \operatorname{sh}^{2 \lambda} y d y\right) \operatorname{sh}^{2 \lambda} x d x \\
& =\int_{\mathbb{R}_{+}} f_{1}(\operatorname{ch} y) \operatorname{sh}^{2 \lambda} y d y \int_{\mathbb{A}} A_{\operatorname{chy} g}^{\lambda} g(\operatorname{ch} x) \operatorname{sh}^{2 \lambda} x d x .
\end{aligned}
$$

Hence by Lemma 3.1 we obtain

$$
\begin{aligned}
& \int_{\mathbb{A}}\left(g \oplus f_{1}\right)(\operatorname{ch} x) \operatorname{sh}^{2 \lambda} x d x \lesssim \int_{0}^{t} g^{*}(\operatorname{ch} u) d u \int_{\mathbb{R}_{+}} f_{1}(\operatorname{ch} y) \operatorname{sh}^{2 \lambda} y d y \\
\leq & \int_{0}^{t} g^{* *}(\operatorname{ch} u) d u \int_{\mathbb{R}_{+}} f_{1}(\operatorname{ch} y) \operatorname{sh}^{2 \lambda} y d y \\
\approx & \left(\int_{E_{t}} f(\operatorname{ch} y) \operatorname{sh}^{2 \lambda} y d y-t f^{*}(\operatorname{ch} t)\right) \int_{0}^{t} g^{* *}(\operatorname{ch} u) d u .
\end{aligned}
$$


Thus by (3.5) we have

$$
\begin{aligned}
\left(g \oplus f_{1}\right)^{* *}(\text { ch } t) & =\frac{1}{t} \sup _{|\mathbb{A}|_{\lambda}=t} \int_{\mathbb{A}}\left(g \oplus f_{1}\right)^{*}(\operatorname{ch} x) \operatorname{sh}^{2 \lambda} x d x \\
& \lesssim\left(f^{* *}(\text { cht })-f^{*}(\text { cht })\right) \int_{0}^{t} g^{* *}(\operatorname{ch} u) d u .
\end{aligned}
$$

Next, estimate $\left(g \oplus f_{2}\right)^{* *}(c h t)$. Taking into account Lemma 3.1 and (3.5) we have

$$
\begin{aligned}
& \left(A_{\text {chy }}^{\lambda} g(\operatorname{ch} x)\right)^{*}(\operatorname{chu}) \leq\left(A_{\text {chy }}^{\lambda} g(\operatorname{ch} x)\right)^{* *}(\operatorname{ch} u) \\
& \lesssim \frac{1}{u} \sup _{|\mathbb{A}|_{t}=u} \int_{\mathbb{A}}\left(A_{\text {chy }}^{\lambda} g(\operatorname{ch} x) \operatorname{sh}^{2 \lambda} y\right)^{*} d y \lesssim g^{* *}(\operatorname{ch} u),
\end{aligned}
$$

hence by Proposition 3.7 we get

$$
\begin{aligned}
& \left(g \oplus f_{2}\right)(\operatorname{ch} x) \leq \int_{0}^{\infty} f_{2}^{*}(\operatorname{ch} u)\left(A_{\text {chy }}^{\lambda} g(\operatorname{ch} x)\right)^{*}(\operatorname{ch} u) d u \leq \int_{0}^{\infty} f_{2}^{*}(\operatorname{ch} u) g^{* *}(\operatorname{ch} u) d u \\
& \lesssim f^{*}(\text { cht }) \int_{0}^{t} g^{* *}(\text { chu }) d u+\int_{t}^{\infty} f^{*}(\text { chu }) g^{* *}(\text { chu }) d u .
\end{aligned}
$$

Consequently by (3.5) we have

$$
\left(g \oplus f_{2}\right)^{* *}(\operatorname{ch} t) \lesssim f^{*}(\operatorname{ch} t) \int_{0}^{t} g^{* *}(\operatorname{ch} u) d u+\int_{t}^{\infty} f^{*}(\operatorname{ch} u) g^{* *}(\operatorname{ch} u) d u
$$

Therefore we obtain (3.8).

Theorem 3.2. If $g \in W L_{r, \lambda}\left(\mathbb{R}_{+}\right), 1<r<\infty$, then

$$
\begin{aligned}
& (f \oplus g)^{*}(\operatorname{ch} t) \leq(f \oplus g)^{* *}(\operatorname{ch} t) \lesssim \frac{r}{r-1}\|g\|_{W L_{r, \lambda}} \\
& \times\left(\left(\operatorname{sh} \frac{t}{2}\right)^{-\frac{1}{r}} \int_{0}^{t} f^{*}(\operatorname{ch} u) d u+\int_{t}^{\infty}\left(\operatorname{sh} \frac{u}{2}\right)^{-\frac{1}{r}} f^{*}(\operatorname{ch} u) d u\right) .
\end{aligned}
$$

Proof. Since $f \in W L_{r, \lambda}\left(\mathbb{R}_{+}\right)$, we have

$$
g^{*}(\operatorname{ch} t) \leq\left(\operatorname{sh} \frac{t}{2}\right)^{-\frac{1}{r}}\|g\|_{W L_{r, \lambda}} g^{* *}(\operatorname{ch} t) \leq \frac{r}{r-1}\left(\operatorname{sh} \frac{t}{2}\right)^{-\frac{1}{r}}\|g\|_{W L_{r, \lambda}} .
$$

Taking into account inequality (3.8) we get the inequality (3.10). 


\section{$4 \mathrm{O}^{\prime}$ Neil inequality for the G-convolution}

In this section we prove $\mathrm{O}^{\prime} \mathrm{Neil}$ inequality for the G-convolution.

Theorem 4.1. 1) Let $1<p<q<\infty, \frac{1}{p^{\prime}}+\frac{1}{q}=\frac{1}{r}, f \in L_{p, \lambda}\left(\mathbb{R}_{+}\right), g \in W L_{r, \lambda}\left(\mathbb{R}_{+}\right)$. Then $f \oplus g \in$ $L_{q, \lambda}\left(\mathbb{R}_{+}\right)$and

$$
\|f \oplus g\|_{L_{q, \lambda}} \leq A\|f\|_{L_{p, \lambda}}\|g\|_{W L_{r, \lambda}}
$$

where $A=2^{\frac{1}{r}} \cdot \frac{r}{r-1}\left(\left(p^{\prime}\right)^{\frac{1}{q}}\left(q^{\prime}\right)^{\frac{1}{p^{\prime}}}+p^{\frac{1}{q}} q^{\frac{1}{p^{\prime}}}\right)$.

2) Let $p=1,1<q<\infty, f \in L_{1, \lambda}\left(\mathbb{R}_{+}\right), g \in W L_{q, \lambda}\left(\mathbb{R}_{+}\right)$. Then $f \oplus g \in W L_{q, \lambda}\left(\mathbb{R}_{+}\right)$and

$$
\|f \oplus g\|_{W L_{q, \lambda}} \lesssim \frac{r}{r-1}\|f\|_{L_{1, \lambda}}\|g\|_{W L_{q, \lambda}}
$$

Proof. 1) Let $f \in L_{p, \lambda}\left(\mathbb{R}_{+}\right), g \in W L_{r, \lambda}\left(\mathbb{R}_{+}\right), 1<p<q<\infty$ and $\frac{1}{r}=\frac{1}{p^{\prime}}+\frac{1}{q}$. From Proposition 3.5 and inequality (3.10) applied Minkowski inequality we get

$$
\begin{gathered}
\|f \oplus g\|_{L_{q, \lambda}}=\left\|(f \oplus g)^{*}\right\|_{L_{q}(0, \infty)} \\
\lesssim \frac{r}{r-1}\|g\|_{W L_{r, \lambda}}\left[\left(\int_{0}^{\infty}\left(\operatorname{sh} \frac{t}{2}\right)^{-\frac{q}{r}}\left(\int_{0}^{t} f^{*}(\operatorname{chu} u d u)^{q} d t\right)^{\frac{1}{q}}\right.\right. \\
\quad+\left(\int_{0}^{\infty}\left(\int_{t}^{\infty}\left(\operatorname{sh} \frac{u}{2}\right)^{-\frac{1}{r}} f^{*}(\operatorname{chu} u d u)^{q} d t\right)^{\frac{1}{q}}\right] \\
\lesssim \frac{2^{\frac{1}{r} r}}{r-1}\|g\|_{W L_{r, \lambda}}\left[\left(\int_{0}^{\infty} t^{-\frac{q}{r}}\left(\int_{0}^{t} f^{*}(\operatorname{ch} u) d u\right)^{q} d t\right)^{\frac{1}{q}}\right. \\
\left.\quad+\left(\int_{0}^{\infty}\left(\int_{t}^{\infty} u^{-\frac{1}{r}} f^{*}(\operatorname{ch} u) d u\right)^{q} d t\right)^{\frac{1}{q}}\right] .
\end{gathered}
$$

By Lemma 2.3, for the validity of the inequality

$$
\left(\int_{0}^{\infty} t^{-\frac{q}{r}}\left(\int_{0}^{t} f^{*}(\operatorname{chu}) d u\right)^{q} d t \leq C_{1}\left(\int_{0}^{\infty} f^{*}(\operatorname{cht} t)^{p} d t\right)^{\frac{1}{p}}\right)
$$

it is necessary and sufficient that

$$
\sup _{t>0}\left(\int_{t}^{\infty} u^{-\frac{q}{r}} d u\right)^{\frac{1}{q}}\left(\int_{0}^{t} d u\right)^{\frac{1}{p^{\prime}}}=\left(\frac{q}{r}-1\right)^{-\frac{1}{q}} \sup _{t>0} t^{\frac{1}{r^{\prime}}-\frac{1}{p}+\frac{1}{q}}<\infty .
$$


Note that $C_{1} \leq\left(\frac{q}{r}-1\right)^{-\frac{1}{q}} q^{\frac{1}{q}}\left(q^{\prime}\right)^{\frac{1}{p^{\prime}}}=\left(p^{\prime}\right)^{\frac{1}{q}}\left(q^{\prime}\right)^{\frac{1}{p^{\prime}}}$ and $\frac{1}{p}-\frac{1}{q}=\frac{1}{r^{\prime}}$. Furthermore, by Lemma 2.4 , for the validity of the inequality

$$
\left(\int_{0}^{\infty}\left(\int_{t}^{\infty} u^{-\frac{1}{r}} f^{*}(\text { chu }) d u\right)^{q} d t\right)^{\frac{1}{q}} \leq C_{2}\left(\int_{0}^{\infty} f^{*}(\text { cht })^{p} d t\right)^{\frac{1}{p}}
$$

it is necessary and sufficient condition that

$$
\sup _{t>0}\left(\int_{0}^{t} d u\right)^{\frac{1}{q}}\left(\int_{t}^{\infty} u^{-\frac{p^{\prime}}{r}} d u\right)^{\frac{1}{p^{\prime}}}=\left(\frac{p^{\prime}}{r}-1\right)^{-\frac{1}{p^{\prime}}} \sup _{t>0} t^{\frac{1}{r^{\prime}}-\frac{1}{p}+\frac{1}{q}}<\infty .
$$

Note that $C_{2} \leq\left(\frac{p^{\prime}}{r}-1\right)^{-\frac{1}{p^{\prime}}} p^{\frac{1}{q}}\left(p^{\prime}\right)^{\frac{1}{p^{\prime}}}=p^{\frac{1}{q}} q^{\frac{1}{p^{\prime}}}$ and $\frac{1}{p}-\frac{1}{q}=\frac{1}{r^{\prime}}$. By using these inequalities and applying Proposition 3.5 we obtain

$$
\|f \oplus g\|_{L_{q, \lambda}} \lesssim A\|f\|_{L_{p, \lambda}}\|g\|_{W L_{q, \lambda}}
$$

where $A=2^{\frac{1}{r}} \cdot \frac{r}{r-1}\left(\left(p^{\prime}\right)^{\frac{1}{q}}\left(q^{\prime}\right)^{\frac{1}{p^{\prime}}}+p^{\frac{1}{q}} q^{\frac{1}{p^{\prime}}}\right)$.

2) Let $p=1,1<q<\infty, f \in L_{1, \lambda}\left(\mathbb{R}_{+}\right)$and $g \in W L_{q, \lambda}\left(\mathbb{R}_{+}\right)$. By inequality (3.10) and Proposition 3.5 we have

$$
\begin{aligned}
& \|f \oplus g\|_{W L_{q, \lambda}}=\sup _{t>0}\left(\operatorname{sh} \frac{t}{2}\right)^{\frac{1}{q}}(f \oplus g)^{*}(\operatorname{ch} t) \\
& \lesssim \frac{r}{r-1}\|g\|_{W L_{q, \lambda}} \sup _{t>0}\left(\operatorname{sh} \frac{t}{2}\right)^{\frac{1}{q}}\left(\left(\operatorname{sh} \frac{t}{2}\right)^{-\frac{1}{r}} \int_{0}^{t} f^{*}(\operatorname{chu} u) d u\right. \\
& \left.\quad+\int_{t}^{\infty}\left(\operatorname{sh} \frac{u}{2}\right)^{-\frac{1}{r}} f^{*}(\operatorname{ch} u) d u\right)=\frac{r}{r-1}\|g\|_{W L_{q, \lambda}} \\
& \quad \times\left(\sup _{t>0} \int_{0}^{t} f^{*}(\operatorname{chu} u) d u+\sup _{t>0}\left(\operatorname{sh} \frac{t}{2}\right)^{\frac{1}{q}} \int_{t}^{\infty}\left(\operatorname{sh} \frac{u}{2}\right)^{-\frac{1}{q}} f^{*}(\operatorname{chu} u) d u\right) \\
& \lesssim \frac{r}{r-1}\|g\|_{W L_{q, \lambda}}\left\|f^{*}\right\|_{L_{1}(0, \infty)} \lesssim \frac{r}{r-1}\|f\|_{L_{1, \lambda}} \cdot\|g\|_{W L_{q, \lambda}} .
\end{aligned}
$$

This complete the proof.

\section{Boundedness of $G$-fractional integral operator in $L_{p, \lambda}$}

We define the G-fractional maximal function by

$$
M_{G}^{\alpha, \lambda} f(\operatorname{ch} x)=\sup _{r>0}\left(\operatorname{sh} \frac{r}{2}\right)^{\alpha-2 \lambda-1} \int_{H(0, r)} A_{c h y}^{\lambda} f(\operatorname{ch} x) \operatorname{sh}^{2 \lambda} y d y,
$$


the G-fractional integral by

$$
J_{G}^{\alpha, \lambda} f(\operatorname{ch} x)=\int_{0}^{\infty} g(\operatorname{ch} y) A_{c h y}^{\lambda} f(\operatorname{ch} x) \operatorname{sh}^{2 \lambda} y d y,
$$

where $H(0, r)=(0, r)$, and

$$
g(\operatorname{ch} y)=\left\{\begin{array}{cc}
\left(\operatorname{sh} \frac{y}{2}\right)^{\alpha-2 \lambda-1}, & 0<y<2 \\
\left(\operatorname{sh} \frac{y}{2}\right)^{\frac{4 \alpha(\alpha-2 \lambda-1)}{2 \lambda+1}}, & 2 \leq y<\infty .
\end{array}\right.
$$

The following relation holds (see [5], Lemma 1.1)

$$
|H(0, r)|_{\lambda}=\int_{0}^{r} \operatorname{sh}^{2 \lambda} t d t \approx \begin{cases}\left(\operatorname{sh} \frac{r}{2}\right)^{2 \lambda+1}, & 0<r<2, \\ \left(\operatorname{ch} \frac{r}{2}\right)^{4 \lambda}, & 2 \leq r<\infty,\end{cases}
$$

and since $\operatorname{sh} t \leq \operatorname{cht} \leq 2 \operatorname{sh} t$ for $t \geq 1$, then

$$
|H(0, r)|_{\lambda} \approx \begin{cases}\left(\operatorname{sh} \frac{r}{2}\right)^{2 \lambda+1}, & 0<r<2 \\ \left(\operatorname{sh} \frac{r}{2}\right)^{4 \lambda}, & 2 \leq r<\infty\end{cases}
$$

We show that $g(\operatorname{ch} x) \in W L_{\frac{2 \lambda+1}{2 \lambda+1-\alpha}, \lambda}\left(\mathbb{R}_{+}\right), 0<\alpha<2 \lambda+1$. Let $0<x<2$. By definition of $g_{*}$, we have

$$
\begin{aligned}
& g_{*}(t)=|\{x \in(0,2):|g(\operatorname{ch} x)|>t\}|_{\lambda} \\
= & \int_{\{x \in(0,2):|g(\operatorname{ch} x)|>t\}} s^{2 \lambda} x d x=\int_{\left\{x \in(0,2):\left(\operatorname{sh}^{2}\right)^{\alpha-2 \lambda-1}>t\right\}} s^{2 \lambda} x d x \\
= & \int_{\left\{x \in(0,2): \operatorname{sh} \frac{x}{2}<t^{\frac{1}{\alpha-2 \lambda-1}}\right\}} \operatorname{sh}^{2 \lambda} x d x=\left|H\left(0, t \frac{1}{\alpha-2 \lambda-1}\right)\right|_{\lambda} .
\end{aligned}
$$

Taking into account (5.4) we have

$$
g_{*}(t) \approx\left(\operatorname{sh} \frac{t}{2}\right)^{-\frac{2 \lambda+1}{2 \lambda+1-\alpha}}, \quad 0<t<2 .
$$

Now let $2 \leq x<\infty$, then from (5.3) we get

$$
\begin{aligned}
& g_{*}(t)=\left|\left\{x \in[2, \infty):|g(\operatorname{ch} x)|_{\lambda}>t\right\}\right|_{\lambda} \\
= & \int_{\{x \in[2, \infty):|g(\operatorname{ch} x)|>t\}} s^{2 \lambda} x d x=\int_{\left\{x \in[2, \infty):\left(\operatorname{sh} \frac{x}{2}\right)^{\frac{4 \lambda(\alpha-2 \lambda-1)}{2 \lambda+1}}>t\right\}} \operatorname{sh}^{2 \lambda} x d x \\
= & \left.\int_{\left\{x \in[2, \infty):\left(\operatorname{sh} \frac{x}{2}\right)<t^{\frac{2 \lambda}{4 \lambda(\alpha-2 \lambda-1)}}\right.}\right\}^{2 \lambda} x d x=\left|H\left(0, t^{\frac{2 \lambda+1}{4 \lambda(\alpha-2 \lambda-1)}}\right)\right|_{\lambda} \\
\approx & \left(\operatorname{sh} \frac{t}{2}\right)^{-\frac{2 \lambda+1}{2 \lambda+1-\lambda}}, \quad 2 \leq t<\infty,
\end{aligned}
$$


with it follows from (5.4).

From (5.5) and (5.6) it follows that

$$
g_{*}(t) \approx\left(\operatorname{sh} \frac{t}{2}\right)^{-\frac{2 \lambda+1}{2 \lambda+1-\alpha}}, 0<t<\infty
$$

From (5.7) we have

$$
\begin{aligned}
g^{*}(\operatorname{cht}) & =\inf \left\{x>0: g_{*}(x) \leq \operatorname{sh} \frac{t}{2}\right\}=\inf \left\{x>0:\left(\operatorname{sh} \frac{x}{2}\right)^{-\frac{2 \lambda+1}{2 \lambda+1-\alpha}} \leq \operatorname{sh} \frac{t}{2}\right\} \\
& =\inf \left\{x>0: \operatorname{sh} \frac{x}{2} \geq\left(\operatorname{sh} \frac{t}{2}\right)^{-\frac{2 \lambda+1-\alpha}{2 \lambda+1}}\right\}=\left(\operatorname{sh} \frac{t}{2}\right)^{-1+\frac{\alpha}{2 \lambda+1}} .
\end{aligned}
$$

Since $p=\frac{2 \lambda+1}{2 \lambda+1-\alpha}$ then from (5.8) we obtain

$$
\|g\|_{W L} \frac{2 \lambda+1}{2 \lambda+1-\alpha}, \lambda=\sup _{t>0}\left(\operatorname{sh} \frac{t}{2}\right)^{\frac{1}{p}} g^{*}(\operatorname{ch} t) \approx\left(\operatorname{sh} \frac{t}{2}\right)^{1-\frac{\alpha}{2 \lambda+1}}\left(\operatorname{sh} \frac{t}{2}\right)^{-1+\frac{\alpha}{2 \lambda+1}}=1 .
$$

By definition of $f^{* *}$, we get

$$
g^{* *}(\operatorname{ch} t)=\frac{1}{t} \int_{0}^{t} g^{*}(\operatorname{ch} x) d x=\frac{1}{t} \int_{0}^{t}\left(\operatorname{sh} \frac{x}{2}\right)^{-1+\frac{\alpha}{2 \lambda+1}} d x \geq\left(\operatorname{sh} \frac{t}{2}\right)^{-1+\frac{\alpha}{2 \lambda+1}}
$$

On the other hand

$$
\begin{aligned}
& g^{* *}(\operatorname{ch} t)=\frac{1}{t} \int_{0}^{t}\left(\operatorname{sh} \frac{x}{2}\right)^{-1+\frac{\alpha}{2 \lambda+1}} d x=\frac{1}{t} \sum_{j=0}^{\infty} \int_{2^{-j-1} t}^{2^{-j} t}\left(\operatorname{sh} \frac{x}{2}\right)^{-1+\frac{\alpha}{2 \lambda+1}} d x \\
\leq & \frac{1}{t} \sum_{j=0}^{\infty}\left(\operatorname{sh} \frac{t}{2^{j+2}}\right)^{-1+\frac{\alpha}{2 \lambda+1}}\left(2^{-j} t-2^{-j-1} t\right)=\sum_{j=0}^{\infty}\left(2^{-j-1} \operatorname{sh} \frac{t}{2}\right)^{-1+\frac{\alpha}{2 \lambda+1}} \cdot 2^{-j-1} \\
= & \left(\operatorname{sh} \frac{t}{2}\right)^{-1+\frac{\alpha}{2 \lambda+1}} \sum_{j=0}^{\infty} 2^{-\frac{\alpha}{2 \lambda+1}(j+1)} \lesssim\left(\operatorname{sh} \frac{t}{2}\right)^{-1+\frac{\alpha}{2 \lambda+1}} .
\end{aligned}
$$

From (5.10) and (5.11) it follows that

$$
g^{* *}(\operatorname{ch} t) \approx g^{*}(\operatorname{ch} t), 0<t<\infty .
$$

Corollary 5.1. Let $0<\alpha<2 \lambda+1$. Then the following inequalities hold

$$
\begin{aligned}
& \left(J_{G}^{\alpha, \lambda} f\right)^{*}(\operatorname{ch} t) \leq\left(J_{G}^{\alpha, \lambda} f\right)^{* *}(\operatorname{ch} t) \\
\lesssim & \left(\operatorname{sh} \frac{t}{2}\right)^{\frac{\alpha}{2 \lambda+1}-1} \int_{0}^{t} f^{*}(\operatorname{ch} u) d u+\int_{t}^{\infty}\left(\operatorname{sh} \frac{t}{2}\right)^{\frac{\alpha}{2 \lambda+1}-1} f^{*}(\operatorname{ch} u) d u .
\end{aligned}
$$


Indeed by the definition of convolution we have

$$
J_{G}^{\alpha, \lambda} f(\operatorname{ch} x)=\int_{\mathbb{R}_{+}} g(\operatorname{ch} y) A_{c h y}^{\lambda} f(\operatorname{ch} x) \operatorname{sh}^{2 \lambda} y d y=(f \otimes g)(\operatorname{ch} x) .
$$

From this, Theorem 3.2 and (5.7) we have (5.13).

Lemma 5.1. Let $0<\alpha<2 \lambda+1$. Then

$$
M_{G}^{\alpha, \lambda} f(\operatorname{ch} x) \leq \frac{2^{2 \lambda+1-\alpha}}{2^{2 \lambda+1-\alpha}-1} J_{G}^{\alpha, \lambda}(|f|)(\operatorname{ch} x) .
$$

Proof. From (5.2) we have

$$
\begin{aligned}
& J_{G}^{\alpha, \lambda}(|f|)(\operatorname{ch} x) \\
= & \sum_{j=-\infty}^{0} \int_{2^{j}}^{2^{j+1}} \frac{A_{c h y}^{\lambda}|f(\operatorname{ch} x)|}{\left(\operatorname{sh} \frac{y}{2}\right)^{2 \lambda+1-\alpha}} \operatorname{sh}^{2 \lambda} y d y+\sum_{j=1}^{\infty} \int_{2^{j}}^{2^{j+1}} \frac{A_{c h y}^{\lambda}|f(\operatorname{ch} x)|}{\left(\operatorname{sh} \frac{y}{2}\right)^{\frac{4 \lambda}{2 \lambda+1}(2 \lambda+1-\alpha)}} \operatorname{sh}^{2 \lambda} y d y \\
= & \sum_{j=-\infty}^{0} J_{G, 1}^{\alpha, \lambda, j}(|f|)(\operatorname{ch} x)+\sum_{j=1}^{\infty} J_{G, 2}^{\alpha, \lambda, j}(|f|)(\operatorname{ch} x),
\end{aligned}
$$

where

$$
\begin{aligned}
& J_{G, 1}^{\alpha, \lambda, j}(|f|)(\operatorname{ch} x)=\int_{2^{j}}^{2^{j+1}} \frac{A_{c h y}^{\lambda}|f(\operatorname{ch} x)|}{\left(\operatorname{sh} \frac{y}{2}\right)^{2 \lambda+1-\alpha}} \operatorname{sh}^{2 \lambda} y d y, \\
& J_{G, 2}^{\alpha, \lambda, j}(|f|)(\operatorname{ch} x)=\int_{2^{j}}^{2^{j+1}} \frac{A_{c h y}^{\lambda}|f(\operatorname{ch} x)|}{\left(\operatorname{sh} \frac{y}{2}\right)^{\frac{4 \lambda}{2 \lambda+1}(2 \lambda+1-\alpha)}} \operatorname{sh}^{2 \lambda} y d y .
\end{aligned}
$$

Further we have

$$
\begin{aligned}
J_{G, 1}^{\alpha, \lambda, j}(|f|)(\operatorname{ch} x) & \geq\left(\operatorname{sh} 2^{j}\right)^{\alpha-2 \lambda-1} \int_{2^{j}}^{2^{j+1}} A_{c h y}^{\lambda}|f(\operatorname{ch} x)| \operatorname{sh}^{2 \lambda} y d y, \\
J_{G, 2}^{\alpha, \lambda, j}(|f|)(\operatorname{ch} x) & \geq\left(\operatorname{sh} 2^{j}\right)^{\frac{4 \lambda}{2 \lambda+1}(\alpha-2 \lambda-1)} \int_{2^{j}}^{2^{j+1}} A_{c h y}^{\lambda}|f(\operatorname{ch} x)| s h^{2 \lambda} y d y \\
& \geq\left(\operatorname{sh} 2^{j}\right)^{\alpha-2 \lambda-1} \int_{2^{j}}^{2^{j+1}} A_{c h y}^{\lambda}|f(\operatorname{ch} x)| \operatorname{sh}^{2 \lambda} y d y .
\end{aligned}
$$

In this way

$$
\begin{aligned}
& J_{G}^{\alpha, \lambda, j}(|f|)(\operatorname{ch} x)=J_{G, 1}^{\alpha, \lambda, j}(|f|)(\operatorname{ch} x)+J_{G, 2}^{\alpha, \lambda, j}(|f|)(\operatorname{ch} x) \\
\geq & \left(\operatorname{sh} 2^{j}\right)^{\alpha-2 \lambda-1} \int_{2^{j}}^{j^{j+1}} A_{c h y}^{\lambda}|f(\operatorname{ch} x)| \operatorname{sh}^{2 \lambda} y d y .
\end{aligned}
$$


Note that

$$
\begin{aligned}
J_{G}^{\alpha, \lambda, j}(|f|)(\operatorname{ch} x) \geq & \left(\operatorname{sh} 2^{j}\right)^{\alpha-2 \lambda-1} \int_{2^{j}}^{j^{j+1}} A_{c h y}^{\lambda}|f(\operatorname{ch} x)| \operatorname{sh}^{2 \lambda} y d y \\
= & \left(\operatorname{sh} 2^{j}\right)^{\alpha-2 \lambda-1} \int_{0}^{2^{j+1}} A_{c h y}^{\lambda}|f(\operatorname{ch} x)| \operatorname{sh}^{2 \lambda} y d y \\
& \quad-\left(\operatorname{sh} 2^{j-1} \cdot 2\right)^{\alpha-2 \lambda-1} \int_{0}^{2^{j}} A_{c h y}^{\lambda}|f(\operatorname{ch} x)| \operatorname{sh}^{2 \lambda} y d y .
\end{aligned}
$$

But

$$
\left(\operatorname{sh} 2^{j-1} \cdot 2\right) \geq\left(2 \operatorname{sh} 2^{j-1}\right)^{2 \lambda+1-\alpha}=2^{2 \lambda+1-\alpha}\left(\operatorname{sh} 2^{j-1}\right)^{2 \lambda+1-\alpha}
$$

then

$$
\left(\operatorname{sh} 2^{j}\right)^{\alpha-2 \lambda-1} \leq 2^{\alpha-2 \lambda-1}\left(\operatorname{sh} 2^{j-1}\right)^{\alpha-2 \lambda-1}
$$

and therefore from (5.15) we have

$$
\begin{aligned}
J_{G}^{\alpha, \lambda, j}(|f|)(\operatorname{ch} x) \geq & \left(\operatorname{sh} 2^{j}\right)^{\alpha-2 \lambda-1} \int_{0}^{2^{j+1}} A_{c h y}^{\lambda}|f(\operatorname{ch} x)| \operatorname{sh}{ }^{2 \lambda} y d y \\
& \quad-2^{\alpha-2 \lambda-1}\left(\operatorname{sh} 2^{j-1}\right)^{\alpha-2 \lambda-1} \int_{0}^{2^{j}} A_{c h y}^{\lambda}|f(\operatorname{ch} x)| \operatorname{sh}^{2 \lambda} y d y \\
\geq & \left(1-2^{\alpha-2 \lambda-1}\right)\left(\operatorname{sh} 2^{j}\right)^{\alpha-2 \lambda-1} \int_{0}^{2^{j+1}} A_{c h y}^{\lambda}|f(\operatorname{ch} x)| \operatorname{sh}^{2 \lambda} y d y .
\end{aligned}
$$

If we take the supremum with respect to $j \in \mathbb{Z}$ in the both sides of the above inequality, then we get

$$
\begin{aligned}
& \sup _{j \in \mathbb{Z}} J_{G}^{\alpha, \lambda, j}(|f|)(\operatorname{ch} x) \\
\geq & \left(1-2^{\alpha-2 \lambda-1}\right) \times \sup _{j \in \mathbb{Z}}\left(\operatorname{sh} 2^{j}\right)^{\alpha-2 \lambda+1} \int_{0}^{2^{j+1}} A_{c h y}^{\lambda}|f(\operatorname{ch} x)| s h^{2 \lambda} y d y .
\end{aligned}
$$

On the other hand, from (5.1) we have

$$
M_{G}^{\alpha, \lambda, j} f(\operatorname{ch} x) \leq \sup _{j \in \mathbb{Z}}\left(\operatorname{sh} 2^{j}\right)^{\alpha-2 \lambda-1} \int_{0}^{2^{j+1}} A_{c h y}^{\lambda}|f(\operatorname{ch} x)| \operatorname{sh}^{2 \lambda} y d y .
$$

Thus, the assertion of Lemma 5.1 follows from (5.14), (5.16) and (5.17).

Corollary 5.2. Let $0<\alpha<2 \lambda+1$, then for $0<t<\infty$

$$
\left(M_{G}^{\alpha, \lambda} f\right)^{*}(\operatorname{ch} t) \leq\left(M_{G}^{\alpha, \lambda} f\right)^{* *}(\operatorname{cht} t)
$$




$$
\lesssim\left(\operatorname{sh} \frac{t}{2}\right)^{\frac{\alpha}{2 \lambda+1}-1} \int_{0}^{t} f^{*}(\operatorname{ch} u) d u+\int_{t}^{\infty}\left(\operatorname{sh} \frac{u}{2}\right)^{\frac{\alpha}{2 \lambda+1}-1} f^{*}(\operatorname{ch} u) d u
$$

Corollary 5.3. Let $0<\alpha<2 \lambda+1$. Then

1) If $1<p<\frac{2 \lambda+1}{\alpha}, f \in L_{p, \lambda}\left(\mathbb{R}_{+}\right)$and $\frac{1}{p}-\frac{1}{q}=\frac{\alpha}{2 \lambda+1}$, then $J_{G}^{\alpha, \lambda} f \in L_{q, \lambda}\left(\mathbb{R}_{+}\right)$and

$$
\left\|J_{G}^{\alpha, \lambda} f\right\|_{L_{q, \lambda}} \lesssim\|f\|_{L_{p, \lambda}}
$$

2) If $p=1, f \in L_{1, \lambda}\left(\mathbb{R}_{+}\right)$and $1-\frac{1}{q}=\frac{\alpha}{2 \lambda+1}$, then $J_{G}^{\alpha, \lambda} f \in W L_{q, \lambda}\left(\mathbb{R}_{+}\right)$and

$$
\left\|J_{G}^{\alpha, \lambda} f\right\|_{W L_{q, \lambda}} \lesssim\|f\|_{L_{p, \lambda}}
$$

Indeed, from (5.5) it follows that

$$
J_{G}^{\alpha, \lambda} f(\operatorname{ch} x)=(f * g)(\operatorname{ch} x)
$$

Suppose $r=\frac{2 \lambda+1}{2 \lambda+1-\alpha}$ and use (5.9) in (4.1) we have the assertion 1) of Corollary 5.3. From the condition 2) it follows that $q=\frac{2 \lambda+1}{2 \lambda+1-\alpha}$. Therefore the assertion 2) follows from (5.9) and (4.2).

Theorem 5.1. Let $0<\alpha<2 \lambda+1$. Then

1) if $1<p<\frac{2 \lambda+1}{\alpha}$, then the condition $\frac{1}{p}-\frac{1}{q}=\frac{\alpha}{2 \lambda+1}$ is necessary and sufficient for the boundedness of $J_{G}^{\alpha, \lambda}$ from $L_{p, \lambda}\left(\mathbb{R}_{+}\right)$to $L_{q, \lambda}\left(\mathbb{R}_{+}\right)$.

2) if $p=1$, then the condition $1-\frac{1}{q}=\frac{\alpha}{2 \lambda+1}$ is necessary and sufficient for the boundedness of $J_{G}^{\alpha, \lambda}$ from $L_{1, \lambda}\left(\mathbb{R}_{+}\right)$to $W L_{q, \lambda}\left(\mathbb{R}_{+}\right)$.

Proof. Sufficiency of Theorem 5.1 follows from Theorem 4.1.

Necessity 1). Let $1<p<\frac{2 \lambda+1}{\alpha}, f \in L_{p, \lambda}\left(\mathbb{R}_{+}\right)$and $J_{G}^{\alpha, \lambda}$ be bounded from $L_{p, \lambda}\left(\mathbb{R}_{+}\right)$to $L_{q, \lambda}\left(\mathbb{R}_{+}\right)$i.e.,

$$
\left\|J_{G}^{\alpha} f\right\|_{L_{q, \lambda}} \lesssim\|f\|_{L_{p, \lambda}}
$$

Moreover assume that $f(x)>0$ is increasing. We define the dilation function $f_{t}(\operatorname{ch} x)$ as follows:

$$
\begin{array}{ll}
f(\operatorname{ch}(\operatorname{th} t) x) \leq f_{t}(\operatorname{ch}(\operatorname{cth} t) x), & 0<t<1, \\
f(\operatorname{ch}(\operatorname{th} t) x) \leq f_{t}(\operatorname{ch}(\operatorname{sh} t) x), & 1 \leq t<\infty .
\end{array}
$$


From (5.18) for $0<t<1$ we have

$$
\begin{aligned}
\left\|f_{t}\right\|_{L_{p, \lambda}} & =\left(\int_{0}^{\infty}\left|f_{t}(\operatorname{ch} x)\right|^{p} \operatorname{sh}^{2 \lambda} x d x\right)^{\frac{1}{p}} \leq\left(\int_{0}^{\infty}|f(\operatorname{ch} t) x|^{p} s h^{2 \lambda} x d x\right)^{\frac{1}{p}} \\
& =(t h t)^{\frac{1}{p}}\left(\int_{0}^{\infty}|f(\operatorname{ch} u)|^{p} \operatorname{sh}^{2 \lambda}(t h t) u d u\right)^{\frac{1}{p}} \\
& \leq(t h t)^{\frac{2 \lambda+1}{p}}\left(\int_{0}^{\infty}|f(\operatorname{ch} u)|^{p} \operatorname{sh}^{2 \lambda} u d u\right)^{\frac{1}{p}} \\
& =(t h t)^{\frac{2 \lambda+1}{p}}\|f\|_{L_{p, \lambda}}=\left(\frac{\operatorname{sh} t}{\operatorname{ch} t}\right)^{\frac{2 \lambda+1}{p}}\|f\|_{L_{p, \lambda}} \\
& \lesssim \frac{1}{(\operatorname{ch} t)^{\frac{2 \lambda+1}{p}-\alpha}}\|f\|_{L_{p, \lambda}} \leq(\operatorname{sh} t)^{\alpha-\frac{2 \lambda+1}{p}}\|f\|_{L_{p, \lambda}}
\end{aligned}
$$

where in the second step we used the transformation $(\operatorname{cth} t) x=u$ and $d x=(t h t) d u$. On the other hand,

$$
\begin{aligned}
\left\|f_{t}\right\|_{L_{p, \lambda}} & =\left(\int_{0}^{\infty}\left|f_{t}(\operatorname{ch} x)\right|^{p} \operatorname{sh}^{2 \lambda} x d x\right)^{\frac{1}{p}} \geq\left(\int_{0}^{\infty}|f(\operatorname{ch}(\operatorname{th} t) x)|^{p} s h^{2 \lambda} x d x\right)^{\frac{1}{p}} \\
& =(\operatorname{cth} t)^{\frac{1}{p}}\left(\int_{0}^{\infty}|f(\operatorname{ch} u)|^{p} \operatorname{sh}^{2 \lambda}(\operatorname{cth} t) u d u\right)^{\frac{1}{p}} \geq(\operatorname{cth} t)^{\frac{2 \lambda+1}{p}}\left(\int_{0}^{\infty}|f(\operatorname{ch} u)|^{p} \operatorname{sh}^{2 \lambda} u d u\right)^{\frac{1}{p}} \\
& =(c t h t)^{\frac{2 \lambda+1}{p}}\|f\|_{L_{p, \lambda}} \geq(\operatorname{cth} t)^{\frac{2 \lambda+1}{p}-\alpha}\|f\|_{L_{p, \lambda}} \geq(\operatorname{sh} t)^{\alpha-\frac{2 \lambda+1}{p}}\|f\|_{L_{p, \lambda}} .
\end{aligned}
$$

Let $1 \leq t<\infty$, then from (5.19) we have

$$
\begin{aligned}
\left\|f_{t}\right\|_{L_{p, \lambda}} & =\left(\int_{0}^{\infty}\left|f_{t}(\operatorname{ch} x)\right|^{p} \operatorname{sh}^{2 \lambda} x d x\right)^{\frac{1}{p}} \geq\left(\int_{0}^{\infty}|f(\operatorname{ch}(\operatorname{th} t) x)|^{p} s h^{2 \lambda} x d x\right)^{\frac{1}{p}} \\
& =(\operatorname{cth} t)^{\frac{1}{p}}\left(\int_{0}^{\infty}|f(\operatorname{ch} u)|^{p} \operatorname{sh}^{2 \lambda}(\operatorname{cth} t) u d u\right)^{\frac{1}{p}} \geq(\operatorname{cth} t)^{\frac{2 \lambda+1}{p}}\left(\int_{0}^{\infty}|f(\operatorname{ch} u)|^{p} \operatorname{sh}^{2 \lambda} u d u\right)^{\frac{1}{p}} \\
& \geq(\operatorname{cth} t)^{\frac{2 \lambda+1}{p}-\alpha}|f|_{L_{p, \lambda}} \geq(\operatorname{sh} t)^{\alpha-\frac{2 \lambda+1}{p}}\|f\|_{L_{p, \lambda}} .
\end{aligned}
$$

On the other hand,

$$
\begin{aligned}
\left\|f_{t}\right\|_{L_{p, \lambda}} & \leq\left(\int_{0}^{\infty}|f(\operatorname{ch}(\operatorname{sh} t) x)|^{p} \operatorname{sh}^{2 \lambda} x d x\right)^{\frac{1}{p}}=(\operatorname{sh} t)^{-\frac{1}{p}}\left(\int_{0}^{\infty}|f(\operatorname{ch} u)|^{p} \operatorname{sh}^{2 \lambda} \frac{u}{s h t} d u\right)^{\frac{1}{p}} \\
& \leq(\operatorname{sh} t)^{-\frac{2 \lambda+1}{p}}\left(\int_{0}^{\infty}|f(\operatorname{ch} u)|^{p} \operatorname{sh}^{2 \lambda} u d u\right)^{\frac{1}{p}} \leq(\operatorname{sh} t)^{\alpha-\frac{2 \lambda+1}{p}}\|f\|_{L_{p, \lambda}} .
\end{aligned}
$$

Combining (5.20)-(5.23) we obtain

$$
\left\|f_{t}\right\|_{L_{p, \lambda}} \approx(\operatorname{sh} t)^{\alpha-\frac{2 \lambda+1}{p}}\|f\|_{L_{p, \lambda}}, \quad 0<t<\infty .
$$


Further from (5.2) for $0<t<1$ from (5.19), we get

$$
\begin{aligned}
\left\|J_{G}^{\alpha, \lambda} f_{t}\right\|_{L_{q, \lambda}} & =\left(\int_{0}^{\infty}\left|J_{G}^{\alpha, \lambda} f_{t}(\operatorname{ch} x)\right|^{q} \operatorname{sh}^{2 \lambda} x d x\right)^{\frac{1}{q}} \leq\left(\int_{0}^{\infty}\left|J_{G}^{\alpha, \lambda} f(\operatorname{ch}(\operatorname{cth} t) x)\right|^{q} s h^{2 \lambda} x d x\right)^{\frac{1}{q}} \\
& =(\operatorname{th} t)^{\frac{1}{q}}\left(\int_{0}^{\infty}\left|J_{G}^{\alpha, \lambda} f(\operatorname{ch} u)\right|^{q} s h^{2 \lambda}(t h t) u d u\right)^{\frac{1}{q}} \\
& \leq(\operatorname{th} t)^{\frac{2 \lambda+1}{q}}\left(\int_{0}^{\infty}\left|J_{G}^{\alpha, \lambda} f(\operatorname{ch} u)\right|^{q} s h^{2 \lambda} u d u\right)^{\frac{1}{q}} \\
& =(\operatorname{th} t)^{\frac{2 \lambda+1}{q}}\left\|J_{G}^{\alpha, \lambda} f\right\|_{L_{q, \lambda}} \leq\left(\frac{\operatorname{ch} t}{\operatorname{sh} t}\right)^{\frac{2 \lambda+1}{q}}\left\|J_{G}^{\alpha, \lambda} f\right\|_{L_{q, \lambda}} \lesssim(\operatorname{sh} t)^{-\frac{2 \lambda+1}{q}}\left\|J_{G}^{\alpha, \lambda} f\right\|_{L_{q, \lambda}},
\end{aligned}
$$

Analogously

$$
\begin{aligned}
\left\|J_{G}^{\alpha, \lambda} f_{t}\right\|_{L_{q, \lambda}} & \geq\left(\int_{0}^{\infty}\left|J_{G}^{\alpha, \lambda} f(\operatorname{ch}(t h t) x)\right|^{q} s h^{2 \lambda} x d x\right)^{\frac{1}{q}} \\
& \geq(\operatorname{cth} t)^{\frac{1}{q}}\left(\int_{0}^{\infty}\left|J_{G}^{\alpha, \lambda} f(\operatorname{ch} u)\right|^{q} \operatorname{sh}^{2 \lambda}(\operatorname{cth} t) u d u\right)^{\frac{1}{q}} \\
& \geq(\operatorname{cth} t)^{\frac{2 \lambda+1}{q}}\left(\int_{0}^{\infty}\left|J_{G}^{\alpha, \lambda} f(\operatorname{ch} u)\right|^{q} \operatorname{sh}^{2 \lambda} u d u\right)^{\frac{1}{q}} \\
& =\left(\frac{\operatorname{ch} t}{\operatorname{sh} t}\right)^{\frac{2 \lambda+1}{q}}\left\|J_{G}^{\alpha, \lambda} f\right\|_{L_{q, \lambda}} \geq(\operatorname{sh} t)^{-\frac{2 \lambda+1}{q}}\left\|J_{G}^{\alpha, \lambda} f\right\|_{L_{q, \lambda}} .
\end{aligned}
$$

Now let $1 \leq t<\infty$. Then from (5.19) we have

$$
\begin{aligned}
\left\|J_{G}^{\alpha, \lambda} f_{t}\right\|_{L_{q, \lambda}} & \geq\left(\int_{0}^{\infty}\left|J_{G}^{\alpha, \lambda} f(\operatorname{ch}(\operatorname{th} t) x)\right|^{q} \operatorname{sh}^{2 \lambda} x d x\right)^{\frac{1}{q}} \\
& =(\operatorname{cth} t)^{\frac{1}{q}}\left(\int_{0}^{\infty}\left|J_{G}^{\alpha, \lambda} f(\operatorname{ch} u)\right|^{q} \operatorname{sh}^{2 \lambda}(\operatorname{cth} t) u d u\right)^{\frac{1}{q}} \\
& \geq(\operatorname{cth} t)^{\frac{2 \lambda+1}{q}}\left\|J_{G}^{\alpha, \lambda} f\right\|_{L_{q, \lambda}} \geq(\operatorname{sh} t)^{-\frac{2 \lambda+1}{q}}\left\|J_{G}^{\alpha, \lambda} f\right\|_{L_{q, \lambda}} .
\end{aligned}
$$

On the other hand

$$
\begin{aligned}
\left\|J_{G}^{\alpha, \lambda} f_{t}\right\|_{L_{q, \lambda}} & \leq\left(\int_{0}^{\infty}\left|J_{G}^{\alpha, \lambda} f(\operatorname{ch}(\operatorname{sh} t) x)\right|^{q} \operatorname{sh}^{2 \lambda} x d x\right)^{\frac{1}{q}} \\
& =(\operatorname{sh} t)^{-\frac{1}{q}}\left(\int_{0}^{\infty}\left|J_{G}^{\alpha, \lambda} f(\operatorname{ch} u)\right|^{q} \operatorname{sh}^{2 \lambda} \frac{u}{s h t} d u\right)^{\frac{1}{q}} \leq(\operatorname{sh} t)^{-\frac{2 \lambda+1}{q}}\left\|J_{G}^{\alpha, \lambda} f\right\|_{L_{q, \lambda}} .
\end{aligned}
$$

Combining (5.25)-(5.28) we obtain

$$
\left\|J_{G}^{\alpha, \lambda} f_{t}\right\|_{L_{q, \lambda}} \approx(\operatorname{sh} t)^{-\frac{2 \lambda+1}{q}}\left\|J_{G}^{\alpha, \lambda} f\right\|_{L_{q, \lambda},}, \quad 0<t<\infty .
$$


Taking into account (5.18) and also (5.29) and (5.24), we get

$$
\begin{gathered}
\left\|J_{G}^{\alpha, \lambda} f\right\|_{L_{q, \lambda}} \approx(\operatorname{sh} t)^{\frac{2 \lambda+1}{q}}\left\|J_{G}^{\alpha, \lambda} f_{t}\right\|_{L_{q, \lambda}} \lesssim(\operatorname{sh} t)^{\frac{2 \lambda+1}{q}}\left\|f_{t}\right\|_{L_{p, \lambda}} \\
\lesssim(\operatorname{sh} t)^{\alpha-\frac{2 \lambda+1}{p}+\frac{2 \lambda+1}{q}}\|f\|_{L_{p, \lambda}}=(\operatorname{sh} t)^{\alpha+(2 \lambda+1)\left(\frac{1}{q}-\frac{1}{p}\right)}\|f\|_{L_{p, \lambda} .}
\end{gathered}
$$

If $\frac{1}{p}-\frac{1}{q}<\frac{\alpha}{2 \lambda+1}$ then for all $f \in L_{p, \lambda}\left(\mathbb{R}_{+}, G\right)$, we have $\left\|J_{G}^{\alpha, \lambda} f\right\|_{L_{q, \lambda}}=0$ as $t \rightarrow 0$.

If $\frac{1}{p}-\frac{1}{q}>\frac{\alpha}{2 \lambda+1}$, then for all $f \in L_{p, \lambda}\left(\mathbb{R}_{+}, G\right)$, we have $\left\|J_{G}^{\alpha, \lambda} f\right\|_{L_{q, \lambda}}=0$ as $t \rightarrow \infty$.

Consequently $\frac{1}{p}-\frac{1}{q}=\frac{\alpha}{2 \lambda+1}$. Again we establish estimate for $\left\|J_{G}^{\alpha, \lambda} f_{t}\right\|_{W L_{q, \lambda}}$. From (5.10) for $0<t<1$ we have

$$
\begin{aligned}
\left\|J_{G}^{\alpha, \lambda} f_{t}\right\|_{W L_{q, \lambda}} & \geq \sup _{r>0} r\left(\int_{\left\{x \in \mathbb{R}_{+}:\left|J_{G}^{\alpha, \lambda} f(\operatorname{ch}(t h t) x)\right|>r\right\}} s^{2 \lambda} x d x\right)^{\frac{1}{q}} \\
& =\sup _{r>0} r(\operatorname{cth} t)^{\frac{1}{q}}\left(\int_{\left\{u \in \mathbb{R}_{+}:\left|J_{G}^{\alpha, \lambda} f(\operatorname{ch} u)\right|>r\right\}} \operatorname{sh}^{2 \lambda}(\operatorname{cth} t) u d u\right)^{\frac{1}{q}} \\
& \geq \sup _{r>0}(c t h t)^{\frac{2 \lambda+1}{q}}\left(\int_{\left\{u \in \mathbb{R}_{+}:\left|J_{G}^{\alpha, \lambda} f(\operatorname{ch} u)\right|>r\right\}} \operatorname{sh}^{2 \lambda} u d u\right)^{\frac{1}{q}} \\
& =(t h t)^{-\frac{2 \lambda+1}{q}}\left\|J_{G}^{\alpha, \lambda} f\right\|_{W L_{q, \lambda}} \geq(\operatorname{sh} t)^{-\frac{2 \lambda+1}{q}}\left\|J_{G}^{\alpha, \lambda} f\right\|_{W L_{q, \lambda}} .
\end{aligned}
$$

On the other hand from (5.19) we get

$$
\begin{aligned}
& \left\|J_{G}^{\alpha, \lambda} f_{t}\right\|_{W L_{q, \lambda}} \leq \sup _{r>0} r\left(\int_{\left\{x \in \mathbb{R}_{+}:\left|J_{G}^{\alpha, \lambda} f(\operatorname{ch}(\operatorname{cth} t) x)\right|>r\right\}} s h^{2 \lambda} x d x\right)^{\frac{1}{q}} \\
& =(t h t)^{\frac{1}{\bar{q}}} \sup _{r>0}\left(\int_{\left\{u \in \mathbb{R}_{+}:\left|J_{G}^{\alpha, \lambda} f(\operatorname{ch} u)\right|>r\right\}} \operatorname{sh}^{2 \lambda}(\text { th } t) u d u\right)^{\frac{1}{q}} \\
& \leq(t h t)^{\frac{2 \lambda+1}{q}}\left\|J_{G}^{\alpha, \lambda} f\right\|_{W L_{q, \lambda}}=\left(\frac{s h t}{c h t}\right)^{\frac{2 \lambda+1}{q}}\left\|J_{G}^{\alpha, \lambda} f\right\|_{W L_{q, \lambda}} \\
& \lesssim \frac{1}{(\operatorname{ch} t)^{\frac{2 \lambda+1}{q}}}\left\|J_{G}^{\alpha, \lambda} f\right\|_{W L_{q, \lambda}} \leq(\operatorname{sh} t)^{-\frac{2 \lambda+1}{q}}\left\|J_{G}^{\alpha, \lambda} f\right\|_{W L_{q, \lambda}}
\end{aligned}
$$


We consider the case $1 \leq t<\infty$. From (5.19) we obtain

$$
\begin{aligned}
\left\|J_{G}^{\alpha, \lambda} f_{t}\right\|_{W L_{q, \lambda}} & \geq \sup _{r>0} r\left(\int_{\left\{x \in \mathbb{R}_{+}:\left|J_{G}^{\alpha, \lambda} f(\operatorname{ch}(\operatorname{th} t) x)\right|>r\right\}} \operatorname{sh}^{2 \lambda} x d x\right)^{\frac{1}{q}} \\
& =(\operatorname{cth} t)^{\frac{1}{q}} \sup _{r>0}\left(\int_{\left\{u \in \mathbb{R}_{+}:\left|J_{G}^{\alpha, \lambda} f(\operatorname{ch} u)\right|>r\right\}} \operatorname{sh}^{2 \lambda}(\operatorname{cth} t) u d u\right)^{\frac{1}{q}} \\
& \geq(\operatorname{cth} t)^{\frac{2 \lambda+1}{q}}\left\|J_{G}^{\alpha, \lambda} f\right\|_{W L_{q, \lambda}} \geq(\operatorname{sh} t)^{-\frac{2 \lambda+1}{q}}\left\|J_{G}^{\alpha, \lambda} f\right\|_{W L_{q, \lambda}} .
\end{aligned}
$$

On the other hand,

$$
\begin{aligned}
\left\|J_{G}^{\alpha, \lambda} f_{t}\right\|_{W L_{q, \lambda}} & \leq \sup _{r>0}\left(\int_{\left\{x \in \mathbb{R}_{+}:\left|J_{G}^{\alpha, \lambda} f(\operatorname{ch}(\operatorname{sh} t) x)\right|>r\right\}} \operatorname{sh}^{2 \lambda} x d x\right)^{\frac{1}{q}} \\
& =(\operatorname{sh} t)^{-\frac{1}{q}} \sup r>0_{r>0}\left(\int_{\left\{u \in \mathbb{R}_{+}:\left|J_{G}^{\alpha, \lambda} f(\operatorname{ch} u)\right|>r\right\}} \operatorname{sh}^{2 \lambda}\left(\frac{u}{\operatorname{sh} t}\right) d u\right)^{\frac{1}{q}} \leq(\operatorname{sh} t)^{-\frac{2 \lambda+1}{q}}\left\|J_{G}^{\alpha, \lambda} f\right\|_{W L_{q, \lambda}} .
\end{aligned}
$$

From (5.30)-(5.33) for all $t>0$, we have

$$
\left\|J_{G}^{\alpha, \lambda} f_{t}\right\|_{W L_{q, \lambda}} \approx(\operatorname{sh} t)^{-\frac{2 \lambda+1}{q}}\left\|J_{G}^{\alpha, \lambda} f\right\|_{W L_{q, \lambda}} .
$$

Let the operator $J_{G}^{\alpha, \lambda}$ be bounded from $L_{1, \lambda}\left(\mathbb{R}_{+}\right)$to $W L_{q, \lambda}\left(\mathbb{R}_{+}\right)$, i.e.,

$$
\left\|J_{G}^{\alpha, \lambda} f\right\|_{W L_{q, \lambda}} \lesssim\|f\|_{L_{1, \lambda}}
$$

then from (5.24) and (5.34), we have

$$
\begin{aligned}
\left\|J_{G}^{\alpha, \lambda} f_{t}\right\|_{W L_{q, \lambda}} & \approx(\operatorname{sh} t)^{\frac{2 \lambda+1}{q}}\left\|J_{G}^{\alpha, \lambda} f\right\|_{W L_{q, \lambda}} \lesssim(\operatorname{sh} t)^{\frac{2 \lambda+1}{q}}\left\|f_{t}\right\|_{L_{1, \lambda}} \\
& \lesssim(\operatorname{sh} t)^{\frac{2 \lambda+1}{q}}(\operatorname{sh} t)^{\alpha-2 \lambda-1}\|f\|_{L_{1, \lambda}}=(\operatorname{sh} t)^{\alpha-(2 \lambda+1)\left(1-\frac{1}{q}\right)}\|f\|_{L_{1, \lambda} .}
\end{aligned}
$$

If $1-\frac{1}{q}<\frac{\alpha}{2 \lambda+1}$, then for all $f \in L_{1, \lambda}\left(\mathbb{R}_{+}, G\right)$, we have $\left\|J_{G}^{\alpha, \lambda} f\right\|_{W L_{q, \lambda}}=0$ as $t \rightarrow 0$.

If $1-\frac{1}{q}>\frac{\alpha}{2 \lambda+1}$, then for all $f \in L_{1, \lambda}\left(\mathbb{R}_{+}, G\right)$, we have $\left\|J_{G}^{\alpha, \lambda} f\right\|_{W L_{q, \lambda}}=0$ as $t \rightarrow \infty$.

Consequently $1-\frac{1}{q}=\frac{\alpha}{2 \lambda+1}$.

Recently, in the work [5] the Gegenbauer-Riesz (G-Riesz) potential

$$
I_{G}^{\alpha} f(\operatorname{ch} x)=\frac{1}{\Gamma\left(\frac{\alpha}{2}\right)} \int_{0}^{\infty}\left(\int_{0}^{\infty} r^{\frac{\alpha}{2}-1} h_{r}(\operatorname{ch} t) d r\right) A_{c h t}^{\lambda} f(\operatorname{ch} x) s h^{2 \lambda} t d t
$$


is introduced, where

$$
h_{r}(\operatorname{ch} t)=\int_{0}^{\infty} e^{-\gamma(\gamma+2 \lambda)} P_{\gamma}^{\lambda}(\operatorname{ch} t)\left(\gamma^{2}-1\right)^{\lambda-\frac{1}{2}} d \gamma, \quad 0<\alpha<2 \lambda+1,
$$

$P_{\gamma}^{\lambda}(c h t)$ is Gegenbauer function. The following inequality (see [5]), Corollary 3.1) is valid

$$
\begin{aligned}
\left|I_{G}^{\alpha} f(\operatorname{ch} x)\right| & \leq \int_{0}^{\infty} \frac{A_{c h t}^{\lambda}|f(\operatorname{ch} x)| \operatorname{sh}^{2 \lambda} t d t}{(\operatorname{sh} t)^{2 \lambda+1-\alpha}} \\
& \leq \int_{0}^{2} \frac{A_{c h t}^{\lambda}|f(\operatorname{ch} x)| \operatorname{sh}^{2 \lambda} t d t}{\left(\operatorname{sh} \frac{t}{2}\right)^{2 \lambda+1-\alpha}}+\int_{2}^{\infty} \frac{A_{c h t}^{\lambda}|f(\operatorname{ch} x)| \operatorname{sh}^{2 \lambda} t d t}{\left(\operatorname{sh} \frac{t}{2}\right)^{\frac{4 \lambda}{2 \lambda+1}(2 \lambda+1-\alpha)}} \\
& =\int_{0}^{\infty} g(\operatorname{ch} y) A_{c h t}^{\lambda}|f(\operatorname{ch} x)| \operatorname{sh}^{2 \lambda} t d t=J_{G}^{\alpha}(|f|)(\operatorname{ch} x) .
\end{aligned}
$$

From this it follows that Corollary 5.3 and Theorem 5.1 are valid for G-Riesz potential $I_{G}^{\alpha} f(\operatorname{ch} x)$.

From Corollary 5.1 and (5.35) we get

Corollary 5.4. Let $0<\alpha<2 \lambda+1$. Then the following inequalities hold

$$
\begin{aligned}
& \left(I_{G}^{\alpha} g\right)^{*}(\operatorname{ch} t) \leq\left(I_{G}^{\alpha} f\right)^{* *}(\operatorname{ch} t) \\
\lesssim & \left(\operatorname{sh} \frac{t}{2}\right)^{\frac{\alpha}{2 \lambda+1}-1} \int_{0}^{t} f^{*}(\operatorname{ch} u) d u+\int_{t}^{\infty}\left(\operatorname{sh} \frac{t}{2}\right)^{\frac{a}{2 \lambda+1}-1} f^{*}(\operatorname{ch} u) d u .
\end{aligned}
$$

Corollary 5.5. Let $0<\alpha<2 \lambda+1$. Then

1) If $1<p<\frac{2 \lambda+1}{\alpha}$, then the condition $\frac{1}{p}-\frac{1}{q}=\frac{\alpha}{2 \lambda+1}$ is necessary and sufficient for the boundedness of $I_{G}^{\alpha}$ from $L_{p, \lambda}\left(\mathbb{R}_{+}\right)$to $L_{q, \lambda}\left(\mathbb{R}_{+}\right)$.

2) If $p=1$, then the condition $1-\frac{1}{q}=\frac{\alpha}{2 \lambda+1}$ is necessary and sufficient for the boundedness of $I_{G}^{\alpha}$ from $L_{1, \lambda}\left(\mathbb{R}_{+}\right)$to $W L_{q, \lambda}\left(\mathbb{R}_{+}\right)$.

Proof. Sufficiency of Corollary 5.5 follows from Theorem 5.1 and Corollary 5.3.

Necessity 1). Let $I_{G}^{\alpha}$ be bounded from $L_{p, \lambda}\left(\mathbb{R}_{+}\right)$to $L_{q, \lambda}\left(\mathbb{R}_{+}\right)$for $1<p<\frac{2 \lambda+1}{\alpha}$, i.e.,

$$
\left\|I_{G}^{\alpha} f\right\|_{L_{q, \lambda}} \lesssim\|f\|_{L_{p, \lambda}} .
$$

Analogously of (5.25) it can be easily shown that

$$
\left\|I_{G}^{\alpha} f_{t}\right\|_{L_{q, \lambda}} \approx(s h t)^{-\frac{2 \lambda+1}{q}}\left\|I_{G}^{\alpha} f\right\|_{L_{q, \lambda}} .
$$


Taking into account (5.25), (5.36) and (5.37), we get

$$
\begin{aligned}
\left\|I_{G}^{\alpha} f\right\|_{L_{q, \lambda}} & \approx(s h t)^{\frac{2 \lambda+1}{q}}\left\|I_{G}^{\alpha} f_{t}\right\|_{L_{q, \lambda}} \lesssim(s h t)^{\frac{2 \lambda+1}{q}}\left\|f_{t}\right\|_{L_{p, \lambda}} \\
& \lesssim(s h t)^{\alpha-\frac{2 \lambda+1}{p}+\frac{2 \lambda+1}{q}}\|f\|_{L_{p, \lambda}}=(s h t)^{\alpha+(2 \lambda+1)\left(\frac{1}{q}-\frac{1}{p}\right)}\|f\|_{L_{p} .}
\end{aligned}
$$

If $\frac{1}{p}-\frac{1}{q}<\frac{\alpha}{2 \lambda+1}$, then for all $f \in L_{p, \lambda}\left(\mathbb{R}_{+}\right)$, we have $\left\|I_{G}^{\alpha} f\right\|_{L_{q, \lambda}}=0$ as $t \rightarrow 0$.

If $\frac{1}{p}-\frac{1}{q}>\frac{\alpha}{2 \lambda+1}$, then for all $f \in L_{p, \lambda}\left(\mathbb{R}_{+}\right)$, we have $\left\|I_{D}^{\alpha} f\right\|_{L_{q, \lambda}}=0$ as $t \rightarrow \infty$.

Therefore we get the equality $\frac{1}{p}-\frac{1}{q}=\frac{\alpha}{2 \lambda+1}$.

2) Suppose that the operator $I_{G}^{\alpha}$ is bounded from $L_{1, \lambda}\left(\mathbb{R}_{+}\right)$to $W L_{q, \lambda}\left(\mathbb{R}_{+}\right)$, i.e.,

$$
\left\|I_{G}^{\alpha} f\right\|_{W L_{q, \lambda}} \lesssim\|f\|_{L_{1, \lambda}} .
$$

From (5.38) we obtain

$$
\left\|I_{G}^{\alpha} f_{t}\right\|_{W L_{q, \lambda}} \approx(\operatorname{sh} t)^{-\frac{2 \lambda+1}{q}}\left\|I_{G}^{\alpha} f\right\|_{W L_{q, \lambda}} .
$$

Now from (5.38), (5.39) and (5.24), we have

$$
\begin{aligned}
\left\|I_{G}^{\alpha} f\right\|_{W L_{q, \lambda}} & \approx(\operatorname{sh} t)^{\frac{2 \lambda+1}{q}}\left\|I_{G}^{\alpha} f_{t}\right\|_{W L_{q, \lambda}} \lesssim(\operatorname{sh} t)^{\frac{2 \lambda+1}{q}}\left\|f_{t}\right\|_{L_{1, \lambda}} \\
& \lesssim(\operatorname{sh} t)^{\frac{2 \lambda+1}{q}}(\operatorname{sh} t)^{\alpha-2 \lambda-1}\left\|\left.f\right|_{L_{1, \lambda}}=(\operatorname{sh} t)^{\alpha+(2 \lambda+1)\left(\frac{1}{q}-1\right)}\right\| f \|_{L_{1, \lambda} .}
\end{aligned}
$$

If $1-\frac{1}{q}<\frac{\alpha}{2 \lambda+1}$, then for all $f \in L_{1, \lambda}\left(\mathbb{R}_{+}\right)$, we have $\left\|I_{G}^{\alpha}\right\|_{W L_{q, \lambda}}=0$ as $t \rightarrow 0$.

If $1-\frac{1}{q}>\frac{\alpha}{2 \lambda+1}$, then for all $f \in L_{1, \lambda}\left(\mathbb{R}_{+}\right)$, we have $\left\|I_{G}^{\alpha}\right\|_{W L_{q, \lambda}}=0$ as $t \rightarrow \infty$.

Therefore we get the equality $1-\frac{1}{q}=\frac{\alpha}{2 \lambda+1}$.

Corollary 5.6. Let $0<\alpha<2 \lambda+1$. Then

1) if $1<p<\frac{2 \lambda+1}{\alpha}$, then the condition $\frac{1}{p}-\frac{1}{q}=\frac{\alpha}{2 \lambda+1}$ is necessary and sufficient for the boundedness of $M_{G}^{\alpha, \lambda}$ from $L_{p, \lambda}\left(\mathbb{R}_{+}\right)$to $L_{q, \lambda}\left(\mathbb{R}_{+}\right)$.

2) If $p=1$, then the condition $1-\frac{1}{q}=\frac{\alpha}{2 \lambda+1}$ is necessary and sufficient for of $M_{G}^{\alpha, \lambda}$ from $L_{1, \lambda}\left(\mathbb{R}_{+}\right)$to $W L_{q, \lambda}\left(\mathbb{R}_{+}\right)$.

Proof. Sufficiency follows from Theorem 5.1 and Lemma 5.1.

Remark 5.1. We note that the results of this paper are analogues in [3].

\section{Acknowledgements}

The authors would like to express their gratitude to the referees for their very valuable comments and suggestions. The research of V.S. Guliyev and E. Ibragimov was partially supported by the grant of 1st Azerbaijan-Russia Joint Grant Competition (Agreement number no. EIF-BGM-4-RFTF-1/2017-21/01/1). 


\section{References}

[1] Gradshteyn I S, Ryzhik M. Table of Integrals, Series and Products, 7 th edition, translated from the Russian, Elsevier / Academic Press, Amsterdam, 2007.

[2] Guliyev V S, Serbetci A,Safarov Z V. Inequality of O'Neil-type for convolutions associated with the Laplace-Bessel differential operator and application. Math Ineq Appl, 2007, 11(1): 99-112.

[3] Guliyev V S, Ibrahimov E. Generalized Gegenbauer shift and some problems of the theory of approximation of functions on the metric of $L_{2, \lambda}$. Trans Natl Acad Sci Azerb Ser Phys-Tech Math Sci, 2015, 35(4): 19-51.

[4] Guliyev V S, Ibrahimov E J. Necessary and sufficient condition for the boundedness of the Gegenbauer-Riesz potential on Morrey spaces. Georgian Math J, 2018, 25(2): 235-248.

[5] Durand L, Fisbane P M, Simmons L M. Exspansion formulas and addition theorems for Gegenbauer functions. J Math Phys, 1976, 17(11): 1933-1948.

[6] Edmunds D E, Ewans W D. Hardy operators, function spaces and embeddings, SpringerVerlag-Berlin Heidelberg, 2004.

[7] Opic B, Kufner A. Hardy-type inequalities. Pitman Research Notes in Mathematics Series 219, Longman Scientific and Technical Harlow 1990.

[8] Kolyada V I. Rearrangements of functions and embedding of anisotropic spaces of Sobolev type. East J Approx, 1999, 4(2): 111-119.

[9] Ibrahimov E J. On Gegenbauer transformation on the half-line. Georgian Math J, 2011, 18: 497-515.

[10] Ibrahimov E J. Maximal operators associated with Gegenbauer expansions on the half-line. I Proc Inst Math Mech Natl Acad Sci Azerb, 2014, 40(1): 104-121.

[11] Ibrahimov E J, Akbulut A. Hardy-Littlewood-Sobolev theorem for Riesz potential generated by Gegenbauer operator. Trans Razmadze Math Inst, 2016, 170(2): 166-199.

[12] Ibrahimov E J, Jafarova S A. On boundedness of the Riesz potential generated by Gegenbauer differential operator on Morrey spaces. Trans Natl Acad Sci Azerb Ser Phys-Tech Math Sci, 2017, 37(4): 49-70.

[13] Maz'ya V G. Sobolev spaces, Springer-Verlag, Princeton Univ. Press, Berlin, 1985.

[14] Stein E M, Weiss G. Introduction to Fourier Analysis on Euclidean Spaces, Princeton Univ. Press, 1971. 